Working Paper 99-86

Economics Series 31

November 1999
Departamento de Economía

Universidad Carlos III de Madrid

Calle Madrid, 126

28903 Getafe (Spain)

Fax (3491) 624-98-75

\title{
SUPPORTING OTHERS AND THE EVOLUTION OF INFLUENCE *
}

\author{
Salvador Barberà and Andrés Perea **
}

\begin{abstract}
In this paper we study environments in which agents can transfer influence to others by supporting them. When planning whom to support, they should take into account the future effect of this, since the receiving agent might use this influence to support others in the future. We show that in the presence of a finite horizon there is an essentially unique optimal support behavior which can be characterized in terms of associated value functions. The analysis of these value functions allows us to derive qualitative properties of optimal support strategies under different specific environments and to explicitly compute the optimal support behavior in some numerical examples. We also investigate the case of an infinite horizon. Examples show that multiple equilibria may appear in this setting, some of wich sustaining a degree of cooperation that would not be possible under a finite horizon.
\end{abstract}

Keywords: Dynamic games, value functions, continuum player set.

Journal of Economic Literature: C61, C72, C73

** Barberà, Departament d`Economia i d`Historia Econòmica, Universitat Autònoma de Barcelona, 08193 Bellaterra-Barcelona, Spain. E-mail: salvador.barbera@uab.es

Perea, Departamento de Economía, Universidad Carlos III de Madrid, calle Madrid 126, 28903 Getafe - Madrid, Spain. E.mail: perea@eco.uc3m.es

* We thank Jordi Massó, Hans Peters, Manuel Santos, Jeroen Swinkels and Fernando VegaRedondo for their very useful suggestiones. 


\section{Introduction}

May it be in my interest to support rivals who can end up helping my friends? Can it be rational to refrain from supporting friends who might end up helping some of my rivals in the future? Questions of this type may arise in many situations where supporting an agent is likely to have important future consequences which I can no longer control once I supported him; the agent I did help to build up strength can use it later on to support others, regardless of my views about such action. Our aim in this paper is to provide a simple model where delay in supporting friends, or deliberate support of opponents can arise as the result of strategic considerations.

The transfer of influence, and the concern for its future consequences, are present in a wide variety of social, economic and political environments. Consider, for instance, a department at a university in which members have preferences over colleagues. The influence of each member depends on the support it gets from colleagues and everybody tries to organize his support in such a way as to help his friends as much as possible. This might lead to situations where members support less preferred colleagues because they anticipate on the fact that these colleagues will support some of their friends. Or, conversely, do not support friends in fear of their future support behavior.

The same kind of reasoning applies to firms that consider transferring technology to other firms. Once the transfer has taken place, the receiving firm might use the technology to transmit it to others in the future. Kotter (1979) stresses the importance of the indirect effect of influence in management, by saying that "a manager can influence someone indirectly by directly influencing others who in turn have impact on that person". Similar phenomena occur when studying the support between political parties in a country, or any other political situation where the political influence depends on the support received from others.

A recent paper by Barberà, Maschler and Shalev (1998) has analyzed a similar phenomenon. Specifically, they consider societies which admit new members by vote among those who are already in. Since newly elected members become future voters, agents in their model are also concerned with the future actions of potential entrants. There is, however, a fundamental difference between our model and that of Barberà et al. Once an agent is elected, he gets the full right to vote. If he is not elected, he has no right at all. Since it is assumed that one vote is enough to be elected, the fact whether my individual voting behavior makes a difference on the final outcome depends heavily on the votes deposited by others. There is thus a basic discontinuity in the transfer of influence from present voters to potential entrants. This discontinuity has a number of important consequences in reality, and it introduces a fundamental multiplicity of equilibia in the theoretical model.

By contrast, our paper concentrates on those cases where the influence gained by agents depends continuously on the support they get from others. Even if support is still modelled as a binary variable, acquired influence grows as one gets supported by more, and more influential people. Influence is here a simple variable, which summarizes possibly quite different and complex abilities of agents. Support is also a summary variable, standing for those social mechanisms which can be used to transfer influence. Whenever our analysis can be used as an approximation, the reader will have to identify these elementary variables with some basic features of the phenomenon at hand. A priori, it is impossible to decide whether the impact of 
support on influence is better modelled as a lump or continuous variable, and reality will always lie in between. We offer our model as a polar case, in which surprisingly simple characterizations of equilibrium behavior can be obtained, and the dynamics of support can be clearly understood.

We propose a stylized dynamic model in which forward looking agents consider the possibility of supporting others, knowing that their support will induce changes in the future distribution of influence, and that they may not control the impact of their initial actions once these have contributed to strengthen other agents. We assume that the decision to support another agent, or not to, is binary; hence, we do not allow for intensities in the support of others.

The presence of every agent $A$ induces (positive or negative) externalities for the other agents and it is assumed that the magnitude of the externality depends proportionally on A's influence. If, for instance, agents $A$ and $B$ are 'friends', $B$ derives a positive utility from A's presence and this utility grows if $A$ becomes more influential. On the other hand, if the effect of $A$ on $B$ were negative, the effect becomes worse if A's influence grows.

The impact of support on the influence distribution among agents is modeled as follows: the influence of an agent increases proportionally to the total influence of agents that support him. This reflects the idea that influential agents have more impact than others since their contribution, when supporting, is more significant. The above described relationship between influence and support allows us to capture two key phenomena. First of all, supporting another agent produces the direct effect of increasing his influence and thereby affecting your own utility (in a positive or negative sense). A second effect is that the receiving agent may use his increase of influence to support other agents in the future. As such, by supporting an agent, one implicitly transmits influence to other agents, too.

The paper proceeds as follows. Section 2 introduces' our model. Section 3 shows that the dynamic game, in which the agents plan their support behavior during a finite number of stages, has an essentially unique subgame perfect equilibrium. Moreover, the equilibrium has an appealingly simple structure: the agents' support behavior at each stage is independent of the history up to that stage and is characterized by value functions which can be computed via some recursive formula working backwards from the final stage. Roughly speaking, the value functions reflect at each stage the future increase of utility that results from supporting a given agent. Put formally, the value $V_{t}(\theta ; x)$ measures the future impact for agent $\theta$ of supporting agent $x$ at stage $t$. The equilibrium states that agent $\theta$ should always support exactly those agents $x$ having positive value.

In Section 4, we introduce a model with a continuum of agents and propose a concept of equilibrium for this setting. The equilibria of the continuum are proven formally to provide good approximations for the equilibria of models with a finlite but large enough set of agents. This allows us to generate exampes for the continuum case (which is computationally much more manageable), and yet be sure that they have approximate counterparts for finite societies, which are indeed those for which our model and equilibrium concepts are more appropriate.

Section 5 is devoted to the analysis of specific models exhibiting interesting dynamic features. We provide examples of societies where agents would systematically avoid to support enemies, and of other where they would transfer influence to some of their enemies. Moreover, we can be very explicit about the features of our model which account for these phenomena.

In Section 6, we provide a sufficient condition on the agents' preferences over others which 
guarantees that support behavior remains constant over time. Section 7 studies optimal support behavior in the presence of an infinite horizon. In contrast to the finite horizon case, multiple subgame perfect equilibria may exist and equilibria need no longer be history independent. We conclude in Section 8.

\section{The Model and an Example}

Before presenting the formal model, we first lay out the intuition behind it. We consider a group of agents in which everybody produces externalities on the well-being of others. There are many different ways in which an agent $\theta$ can affect the utility of others: agents can simply derive (positive or negative) utility from $\theta$ 's presence or $\theta$ could take certain decisions which affect the well-being of other agents. In this model, we abstract away from the underlying factors inducing these externalities and simply assume that an agent's utility depends on the presence of others. As such, these utilities should be viewed as the final result of some possibly complicated interaction process among agents.

A key factor in the model is the fact that the externality produced by agent $\theta$ on the utility of others is not exogenous but varies over time, depending on actions of other agents. More precisely, we assume that this externality (the intensity with which it affects others) is proportional to some factor which we call the "influence" of $\theta$. The word "influence" should thus be read as the impact that $\theta$ has on the utility of others.

We realize that in reality an agent's influence depends on many different factors: too many to be captured within one model. Among these factors, we filter an important one, namely the support an agent gets from other agents. Here, support should be read in a broad sense since it could take many forms, varying from financial or technological support between firms to political support between parties. Important is that an agent's influence changes over time due to the support it gets from others. On the other hand, this agent can use his influence to support others in the future and thereby increase the influence of other agents. It is this strong mutual relationship between supporting others on one hand and the influence of agents on the other hand that is at the very heart of our model. Formally, the model is as follows.

The group of agents is given by a countable space $\Theta=\left\{\theta_{1}, \theta_{2}, \ldots\right\}$. At stages $t=1, \ldots, T$ agents $\theta \in \Theta$ simultaneously choose to support a set $S_{t}(\theta) \subset \Theta$ of agents. Support is therefore modeled as a binary variable: one can either support an agent or not support him. We do not allow for different levels of support.

Before agents come into action there is an initial distribution of influence among the agents which is given by a strictly positive measure ${ }^{1} \pi_{0}$ on $\Theta$. The support behavior of agents induces changes in the influence distribution. We assume that the influence of an agent increases proportionally to the total influence of agents that support him in this stage. This means that supporting an agent will always contribute to his strength, and this contribution will be more significant if the supporting agent is more influential. Formally, let $\pi_{t}$ be the function representing the influence distribution among agents at stage $t$. The evolution of influence over time is

\footnotetext{
${ }^{1} \mathrm{~A}$ measure on a countable space is strictly positive if it assigns strictly positive mass to every single point.
} 
given by the recursive equation

$$
\pi_{t}(\theta)=\pi_{t-1}(\theta)+\rho(\theta) \cdot \sum_{x \in \Theta: \theta \in S_{t}(x)} \pi_{t-1}(x)
$$

where $\rho$ is some strictly positive measure on $\theta^{2}$ Here, $\rho(\theta)$ can be viewed as a measure for $\theta^{\prime} \mathrm{s}$ sensitivity to support. If, for instance, $\rho(\theta)$ is high, supporting $\theta$ contributes considerably to his influence whereas a low $\rho(\theta)$ allows only for a small increment of his influence.

The utility for $\theta$ at stage $t$ is given by

$$
U_{t}(\theta)=\sum_{x \in \Theta} u(\theta ; x) \pi_{t}(x)
$$

So, $\theta$ 's utility depends linearly on the influence of each agent $x$ and the coefficient $u(\theta ; x)$ can be interpreted as some normalized utility for agent $\theta$ induced by the presence of $x$. The function $u: \Theta \times \Theta \rightarrow \mathbb{R}$ generating these utility coefficients is called a utility profile. If $u(\theta ; x) \geq 0$, then $x$ 's presence is good for $\theta$ and we say that $x$ is a friend of $\theta$. If $u(\theta ; x) \leq 0$, agent $x$ is called an enemy of $\theta$. By comparing the magnitudes of $u(\theta ; x)$, one can distinguish between big friends and small friends, big enemies and small enemies.

In particular, $\theta^{\prime}$ 's utility increases if his friends become more influential whereas his utility decreases if the influence of enemies becomes larger. The overall utility for $\theta$ is equal to

$$
U(\theta)=\sum_{t=1}^{T} \lambda_{t} U_{t}(\theta),
$$

where $\lambda_{t}$ are non-negative constants reflecting the relative contributions of stage utilities to the total utility. Canonical time separable utilities such as average utility and discounted utility can be modeled by choosing $\lambda_{t}=1 / T$ and $\lambda_{t}=\delta^{t}$ respectively.

After each stage, all agents observe the groups of agents $S_{t}(\theta)$ supported by the others. A strategy for agent $\theta$ is to choose at every stage $t$ a set $S_{t}\left(\theta \mid h_{t}\right)$ of agents to be supported by him after any possible history $h_{t}$ at stage $t$. Here, a history $h_{t}$ is a sequence $\left(S_{k}(\theta)\right)_{k \leq t-1, \theta \in \Theta}$ consisting of groups $S_{k}(\theta)$ supported by agents at previous stages.

The quadruple $\Sigma=\left(\Theta, u, \rho, \pi_{0}\right)$ consisting of the set of agents, the utility profile, the support sensitivity measure and the initial distribution of influence, is called a society. The dynamic game induced by this society is denoted by $\Gamma(\Sigma)$.

In order to illustrate the different ingredients of our model, consider the following example.

Example 2.1. A department at a university has three research areas: $A, B$ and $C$. During two consecutive years, the department will hire new scientists in these areas. At times $t=1,2$, the current members must decide about the number of people to be hired in each area. To this purpose, the department has agreed upon the following rule: each member can vote for (one

\footnotetext{
${ }^{2}$ Here, we implicitly assume that $\sum_{x \in \Theta: \theta \in S_{t}(x)} \pi_{t-1}(x)<\infty$.
} 
or more) areas and the number of new positions in a given area is proportional to the number of votes this area gets, with proportionality coefficient 0.5 . For sake of symmetry, assume that members belonging to the same area have identical preferences and will therefore vote identically. Each area $\theta \in\{A, B, C\}$ can therefore be regarded as an individual agent. Let $\pi_{t}(\theta)$ be the number of members working in area $\theta$ at period $t$ and $S_{t}(\theta) \subset\{A, B, C\}$ the set of areas voted for by $\theta$ at the beginning of time $t$. According to the voting rule,

$$
\pi_{t}(\theta)=\pi_{t-1}(\theta)+0.5 \sum_{\substack{x \in\{A, B, C\} \\ \theta \in S_{t}(x)}} \pi_{t-1}(x)
$$

for $t=1,2 .^{3}$ Here, $\pi_{0}$ denotes the area configuration in the department before the voting procedure starts. Suppose, for instance, that the initial area configuration is given by $\pi_{0}=$ $(10,5,15)$, meaning that there are 10 area $A$ people, 5 area $B$ people and 15 area $C$ people in the department before voting starts.

In this context, the numerical representation $\pi_{t}(\theta)$ of area $\theta$ at time $t$ can be regarded as the "influence" of this area within the department. An area $\theta$ can transfer influence to another area $x$ (or, support $x$ ) by voting for $x$. The increase of $x$ 's influence is proportional to the aggregate influence of areas that support it.

Suppose that the utility for a member of area $\theta$ at time $t$ is given by

$$
U_{t}(\theta)=u(\theta ; A) \pi_{t}(A)+u(\theta ; B) \pi_{t}(B)+u(\theta ; C) \pi_{t}(C)
$$

where $u(\theta ; x)$ reflect agent $\theta$ 's preferences over the different areas. The (positive or negative) effect of an area $x$ on a member of area $\theta$ is thus proportional to the number of department members currently working in area $x$. Let the utilities $u(\theta ; x)$ be given by the following matrix:

\begin{tabular}{|c|c|c|c|}
\hline & $A$ & $B$ & $C$ \\
\hline$A$ & 2 & 1 & -6 \\
\hline$B$ & -4 & 3 & 1 \\
\hline$C$ & 2 & -1 & 6 \\
\hline
\end{tabular}

The aggregate utility for agent $\theta$ during the two periods is given by

$$
U(\theta)=U_{1}(\theta)+U_{2}(\theta)
$$

The two-stage game defined above yields a unique subgame perfect voting behavior, given by the following table:

\begin{tabular}{|c|c|c|}
\hline & $\bar{t}=1$ & $t=2$ \\
\hline$A$ & $\{A\}$ & $\{\overline{A, B\}}$ \\
\hline$B$ & $\{B, C\}$ & $\{\overline{B, C}\}$ \\
\hline$C$ & $\{A, \bar{B}, C\}$ & $\{A, C\}$ \\
\hline
\end{tabular}

\footnotetext{
${ }^{3}$ In case $\pi_{t}(\theta)$ is not integer, the department will hire part time workers. If, for instance, a scientist is hired on a $50 \%$ basis, his vote will only count for $50 \%$.
} 
This equilibrium behavior is independent of the initial distribution of areas $\pi_{0}$. Moreover, the behavior specified at stage 2 is optimal for any combination of votes that ocurred at stage 1 .

At the last stage, every area simply votes for its preferred areas (areas $x$ giving positive utility $u(\theta ; x)$ ), since there are no future consequences which have to be taken into account. At the first stage, each area faces a tradeoff between the immediate consequence of voting for an area (department hires new people in this area) and its future consequences (newly admitted members will have the right to vote at stage 2). For instance, area $A$ will not vote for $B$ (despite its positive direct effect) at stage 1 since it knows that $B$ will vote for $C$ at stage 2 , inducing a negative effect which outrules the positive effect at stage 1 . On the other hand, $C$ will vote for $B$ at the first stage (despite the negative direct effect) since it knows that $B$ will vote for $C$ at the next stage, compensating the negative effect at the first stage.

\section{Optimal Support Behavior}

In the example above, we have seen that there is a unique subgame perfect equilibrium in the twostage game. In this section, we show that this is not a coincidence: every dynamic game induced by the model has an essentially unique subgame perfect equilibrium. By 'essentially unique', we mean that it is unique up to ties occurring when an agent $\theta$ is indifferent between supporting another agent $x$ or not. Moreover, the subgame perfect equilibrium has a very simple structure. First of all, the support behavior at every stage is history independent, hence an agent need not keep track of all the groups that have been supported at previous stages. Another property is that the equilibrium behavior does not depend on the initial influence distribution. Finally, the equilibrium is characterized by a sequence of value functions (one for every stage) which can be computed recursively, starting at the final stage. Intuitively, a value function at a given stage assigns to every pair $(\theta ; x)$ a measure for the future increase in $\theta$ 's utility which results from supporting $x$ at this stage. The equilibrium states that at every stage agent $\theta$ should exactly support those agents $x$ that have positive value.

In order to make our statement precise, we need some more definitions. A strategy profile $S$ (prescribing a strategy for each agent) is called a subgame perfect equilibrium if at any stage $t$ and after any history $h_{t}$, the group of agents $S_{t}\left(\theta \mid h_{t}\right)$ supported by agent $\theta$ maximizes $\theta$ 's continuation payoff, given the behavior of other agents at this stage and the behavior of all agents at future stages.

In general, there may exist multiple subgame perfect equilibria. However, multiplicity is solely due to the fact that at some points in the game an agent $\theta$ is indifferent between supporting another agent $x$ and not supporting him. In this case, both supporting $x$ and not supporting $x$ can be part of a subgame perfect equilibrium. Since this kind of indifferences leads to subgame perfect equilibria which are essentially identical, we introduce a tie braking rule which states that in case of indifference, the agent $x$ should be supported. ${ }^{4}$ Hence, the agent should always support the largest group that maximizes his continuation payoff. Subgame perfect equilibria satisfying this tie breaking rule are called maximal subgame perfect equilibria.

\footnotetext{
${ }^{4}$ The tie breaking rule is rather arbitrary. One could also require that in case of indifference, the agent $x$ should not be supported. However, this would lead to essentially identical results throughout the paper.
} 
For every stage $t$, let the value function $V_{t}: \Theta \times \Theta \rightarrow \mathbb{R}$ be given by the recursive formula

$$
\begin{aligned}
V_{T}(\theta ; x) & =\lambda_{T} u(\theta ; x) \text { and } \\
V_{t}(\theta ; x) & =\lambda_{t} u(\theta ; x)+V_{t+1}(\theta ; x)+\sum_{y: V_{t+1}(x ; y) \geq 0} V_{t+1}(\theta ; y) \cdot \rho(y)
\end{aligned}
$$

for all $t<T$. The recursive equation for the value functions can be seen as a multi-agent variant of the Hamilton-Jacobi-Bellman equation in dynamic programming. It states that the value $V_{t}(\theta ; x)$ for $\theta$ of supporting $x$ at stage $t$ can be decomposed into an immediate effect $\lambda_{t} u(\theta ; x)$ and a future effect, given by the last two terms. The future effect consists of the corresponding value $V_{t+1}(\theta ; x)$ at the next stage and an interaction effect represented by the sum. The interaction effect describes the consequences for $\theta$ of having $x$ support other agents in the future: an effect which does not occur in 'standard' one-agent dynamic programming. 5

Although the use of value functions in dynamic one-person decision problems is widely spread (see, for instance, Streufert, 1990, and Kreps and Porteus, 1979, among many, many others), its application to dynamic games is in general complicated. This is due to the fact that for a given player, the value of every decision depends crucially on the future behavior of the other players. Hence, one has to define a value function for each player and these value functions interact at every stage of the game. However, using value functions to solve a dynamic game by backward induction is by no means new. Gomes et al. (1999), for example, introduce a backward induction function to solve a non-cooperative bargaining game with finite horizon. This backward induction function can be viewed as a value function since at any stage it captures the future effect of proposing a certain payoff vector.

Theorem 3.1. For every society $\Sigma$, the game $\Gamma(\Sigma)$ contains a unique maximal subgame perfect equilibrium, which is the history independent strategy profile $S$ given by

$$
S_{t}\left(\theta \mid h_{t}\right)=\left\{x \mid V_{t}(\theta ; x) \geq 0\right\}
$$

for all $t, h_{t}$ and $\theta$.

Proof. Let $S$ be a strategy profile. We show by induction on $t$ that the unique maximal subgame perfect behavior at stage $t$ is given by $S_{t}\left(\theta \mid h_{t}\right)=\left\{x \mid V_{t}(\theta ; x) \geq 0\right\}$.

We start at the final stage $T$. Let $h_{T}$ be an arbitrary but fixed history up to stage $T$ and let $\theta$ be an agent. Independently of the other agents' behavior at this stage, it is optimal for $\theta$ to support all those agents $x$ with $\lambda_{T} u(\theta ; x)>0$ and not to support any agent $x$ with $\lambda_{T} u(\theta ; x)<0$. Maximality requires $\theta$ to support all those agents with $u(\theta ; x)=0$ and therefore it follows that $S_{T}\left(\theta \mid h_{T}\right)=\left\{x \mid \lambda_{T} u(\theta ; x) \geq 0\right\}=\left\{x \mid V_{T}(\theta ; x) \geq 0\right\}$. Since this holds for any history $h_{T}$ and any agent $\theta$ the statement is true at the final stage.

Now, let $\tau<T$ and let $S_{t}(\theta)=\left\{x \mid V_{t}(\theta ; x) \geq 0\right\}$ be the unique maximal subgame perfect support behavior at stage $t$ for all $t \geq \tau$ and all histories $h_{t}$. Let $h_{\tau}$ be an arbitrary but fixed history at the beginning of stage $\tau$. First, we need some definitions and technical observations.

\footnotetext{
${ }^{5}$ In one-person dynamic programming with time separable utility functions, the value function at stage $t$ is simply the sum of the immediate effect and the value at the next stage.
} 
For all $t \geq \tau$, let the functions $s_{t}: \Theta \times \Theta \rightarrow\{0,1\}$ be defined by

$$
s_{t}(\theta ; x)= \begin{cases}1, & \text { if } x \in S_{t}(\theta) \\ 0, & \text { otherwise }\end{cases}
$$

Let $\pi_{t}, t \geq \tau$, be the unique influence distribution at stage $t$, given that we start with history $h_{\tau}$ at stage $\tau$ and given that agents act according to $S$ at future stages. The evolution of $\pi_{t}$ is given by

$$
\begin{aligned}
\pi_{t}(x) & =\pi_{t-1}(x)+\rho(x) \cdot \sum_{\theta \in \Theta: x \in S_{t}(\theta)} \pi_{t-1}(\theta) \\
& =\pi_{t-1}(x)+\sum_{\theta \in \Theta} s_{t}(\theta ; x) \pi_{t-1}(\theta) \rho(x)
\end{aligned}
$$

for all $x \in \Theta$.

For a measure $\mu$ on $\Theta$ and a function $f: \Theta \times \Theta \rightarrow \mathbb{R}$ let the measure $\bar{f} \mu$ on $\Theta$ be given by

for all $x$. Hence, by definition,

$$
\bar{f} \mu(x)=\sum_{\theta} f(\theta ; x) \mu(\theta) \rho(x)
$$

$$
\pi_{t}=\pi_{t-1}+\bar{s}_{t} \pi_{t-1}=\left(I+\bar{s}_{t}\right) \pi_{t-1}
$$

where $I$ is the identity operator on measures.

Let $f, g: \Theta \times \Theta \rightarrow \mathbb{R}$. We define the function $\tilde{f} g: \Theta \times \Theta \rightarrow \mathbb{R}$ by

$$
\tilde{f} g(\theta ; x)=\sum_{y} g(\theta ; y) f(x ; y) \rho(y)
$$

Lemma 3.2. Let $f, g: \Theta \times \Theta \rightarrow \mathbb{R}$ and $\mu$ a measure on $\Theta$. Then, it holds that

$$
\sum_{x} g(\theta ; x) \bar{f} \mu(x)=\sum_{x} \tilde{f} g(\theta ; x) \mu(x)
$$

The proof of this lemma can be found in the appendix.

Let $U_{t}(\theta)=\sum_{x} u(\theta ; x) \pi_{t}(x)$ be the utility for agent $\theta$ at stage $t$ generated by $h_{\tau}$ and the strategy profile $S$.

Lemma 3.3. For every $t \geq \tau$ it holds that

$$
U_{t}(\theta)=\sum_{x}\left[\prod_{l=\tau}^{t}\left(I+\tilde{s}_{l}\right) u\right](\theta ; x) \pi_{\tau-1}(x) .
$$


Here, by $\prod_{l=\tau}^{t}\left(I+\tilde{s}_{l}\right) u$ we mean the function obtained from $u$ by succesively applying the operators $\left(I+\tilde{s}_{\tau}\right),\left(I+\tilde{s}_{\tau+1}\right), \ldots,\left(I+\tilde{s}_{t}\right)$.

The proof of this lemma can be found in the appendix.

Lemma 3.3 implies that

$$
\begin{aligned}
\sum_{t=\tau}^{T} \lambda_{t} U_{t}(\theta) & =\sum_{x}\left[\sum_{t=\tau}^{T} \lambda_{t} \prod_{l=\tau}^{t}\left(I+\tilde{s}_{l}\right) u\right](\theta ; x) \pi_{\tau-1}(x) \\
& =\sum_{x}\left[\left(I+\tilde{s}_{\tau}\right)\left(\lambda_{\tau} u+\sum_{t=\tau+1}^{T} \lambda_{t} \prod_{l=\tau+1}^{t}\left(I+\tilde{s}_{l}\right) u\right](\theta ; x) \pi_{\tau-1}(x)\right.
\end{aligned}
$$

Let the function $w_{\tau}: \Theta \times \Theta \rightarrow \mathbb{R}$ be given by

$$
w_{\tau}(\theta ; x)=\left[\lambda_{\tau} u+\sum_{t=\tau+1}^{T} \lambda_{t} \prod_{l=\tau+1}^{t}\left(I+\tilde{s}_{l}\right) u\right](\theta ; x) .
$$

Hence,

$$
\begin{aligned}
\sum_{t=\tau}^{T} \lambda_{t} U_{t}(\theta) & =\sum_{x}\left(I+\tilde{s}_{\tau}\right) w_{\tau}(\theta ; x) \pi_{\tau-1}(x) \\
& =\sum_{x} w_{\tau}(\theta ; x) \pi_{\tau-1}(x)+\sum_{x} \tilde{s}_{\tau} w_{\tau}(\theta ; x) \pi_{\tau-1}(x)
\end{aligned}
$$

By definition of $\tilde{s}_{\tau} w_{\tau}$ we have

$$
\begin{aligned}
\sum_{x} \tilde{s}_{\tau} w_{\tau}(\theta ; x) \pi_{\tau-1}(x)= & \sum_{x} \sum_{y} w_{\tau}(\theta ; y) s_{\tau}(x ; y) \rho(y) \pi_{\tau-1}(x) \\
= & \pi_{\tau-1}(\theta) \sum_{y} w_{\tau}(\theta ; y) s_{\tau}(\theta ; y) \rho(y) \\
& +\sum_{x \neq \theta} \sum_{y} w_{\tau}(\theta ; y) s_{\tau}(x ; y) \rho(y) \pi_{\tau-1}(x) .
\end{aligned}
$$

Since, by the induction assumption, $S$ is history independent at stages $t \geq \tau$, it follows that

$$
\sum_{x} w_{\tau}(\theta ; x) \pi_{\tau-1}(x)
$$

and

$$
\sum_{x \neq \theta} \sum_{y} w_{\tau}(\theta ; y) s_{\tau}(x ; y) \rho(y) \pi_{\tau-1}(x)
$$

do no depend on $S_{\tau}(\theta)$. Combining equations (3.1) and (3.2) leads therefore to the following conclusion. 
Lemma 3.4. At stage $\tau$ it holds that

$$
\sum_{t=\tau}^{T} \lambda_{t} U_{t}(\theta)=C+\pi_{\tau-1}(\theta) \sum_{y} w_{\tau}(\theta ; y) s_{\tau}(\theta ; y) \rho(y)
$$

where $C$ is an expression that does not depend on $S_{\tau}(\theta)$.

It can be checked immediately that the functions $w_{t}: \Theta \times \Theta \rightarrow \mathbb{R}$ (for $t \geq \tau$ ) are given by the following recursive formula: $w_{T}(\theta ; x)=\lambda_{T} u(\theta ; x)$ and

By definition,

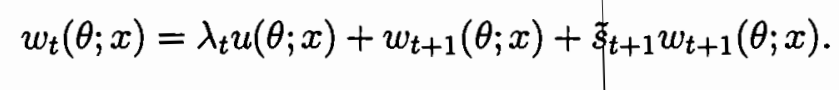

$$
\begin{aligned}
\tilde{s}_{t+1} w_{t+1}(\theta ; x) & =\sum_{y} w_{t+1}(\theta ; y) s_{t+1}(x ; y) \rho(y)=\sum_{y \in S_{t+1}(x)} w_{t+1}(\theta ; y) \rho(y) \\
& =\sum_{y: V_{t+1}(x ; y) \geq 0} w_{t+1}(\theta ; y) \rho(y),
\end{aligned}
$$

using the induction assumption that $S_{t+1}(x)=\left\{y \mid V_{t+1}(x ; y) \geq 0\right\}$. Hence,

$$
\begin{aligned}
w_{T}(\theta ; x) & =\lambda_{T} u(\theta ; x)=V_{T}(\theta ; x) \text { and } \\
w_{t}(\theta ; x) & =\lambda_{t} u(\theta ; x)+w_{t+1}(\theta ; x)+\sum_{y: V_{t+1}(x ; y) \geq 0} w_{t+1}(\theta ; y) \rho(y)
\end{aligned}
$$

for all $t \geq \tau$. This means that the functions $w_{t}, t \geq \tau$, are given by the same recursive formula as the value functions $V_{t}, t \geq \tau$. However, this implies that $w_{t}(\theta ; x)=V_{t}(\theta ; x)$ for all $t \geq$ $\tau$.

Combining this result with Lemma 3.4 leads to the observation that the continuation payoff is equal to

$$
\begin{aligned}
\sum_{t=\tau}^{T} \lambda_{t} U_{t}(\theta) & =C+\pi_{\tau-1}(\theta) \sum_{y} V_{\tau}(\theta ; y) s_{\tau}(\theta ; y) \rho(y) \\
& =C+\pi_{\tau-1}(\theta) \sum_{y \in S_{\tau}(\theta)} V_{\tau}(\theta ; y) \rho(y)
\end{aligned}
$$

where $C$ is an expression which does not depend on $S_{\tau}(\theta)$.

Since $\pi_{0}(\theta)>0$ and $\pi_{t}$ is non-decreasing over time, we have $\pi_{\tau-1}(\theta)>0$. As such, maximizing the continuation payoff at stage $\tau$ is a matter of choosing $S_{\tau}(\theta)$ such that

$$
\sum_{y \in S_{\tau}(\theta)} V_{\tau}(\theta ; y) \rho(y)
$$

is maximal. Since $S$ is assumed to be a maximal subgame perfect equilibrium, $S_{\tau}(\theta)=\{x \mid$ $\left.V_{\tau}(\theta ; x) \geq 0\right\}$. This holds for any history $h_{\tau}$, hence $S_{\tau}\left(\theta \mid h_{\tau}\right)=\left\{x \mid V_{\tau}(\theta ; x) \geq 0\right\}$ for all $h_{\tau}$. This completes the proof of the theorem. 


\section{Limit Behavior in Continuum Societies}

One of the aims of this paper is to illustrate the phenomenon of postponement or advancement of support for specific classes of problems, as a means to understand what features in the preferences of agents are crucial in generating different types of behavior. Yet, finding numerical examples, or interesting classes of parametrized problems, is not an easy task. This task could be facilitated if we could work with continuous variables; that is, if we had a model with a continuum of agents over which other agents could then have preferences, representable by continuous utility functions. The use of models with a continuum of agents has a long tradition in economics and political science (see, for instance, Schmeidler (1973) and Aumann (1964) among many others) and we would not elaborate much on it, were it not that, in our case, a simple extrapolation of concepts without some further interpretation could be misleading. We shall work, indeed, with a continuum of agents, each of which will have mass zero. Hence, as in standard models, changes in the support behavior of a single agent will have no influence at all on the overall outcome. Moreover, and this is a crucial difference with, say, Aumann's (1964) model of a competitive economy, any individual in the continuum society will be indifferent among all types of support behavior that are available to him. This follows from the fact that support is the agent's only choice variable, and yet it does affect his utility. (This in contrast with a consumer's behavior in the continuum; he cannot affect prices, but still derives different utilities from different consumption plans.)

Because of that, our model of a continuum society is no longer a game in any proper sense, and equilibria for this model can hardly be justified per se. Instead, we use a continuum society as an approximation of large discrete societies, and equilibrium behavior is defined in terms of the discrete societies approximating the continuum. As we shall see, both the model and the equilibrium notion proposed will prove to be useful and to provide a well grounded method to construct examples which have direct bearing for the understanding of discrete societies. To do that, we now discuss the model, the equilibrium concept, and their relationships with large discrete societies.

\subsection{Limit Equilibria}

Before stating the formal definition our equilibrium concept, let us briefly sketch the idea. Our starting point is a society $\Sigma$ with a continuum set of agents $\Theta$. For every $n \in \mathbb{N}$ we select a discrete set $\Theta^{n}$ of representatives from $\Theta$ such that $\Theta^{n}$ becomes dense in $\Theta$. To every $\Theta^{n}$, there corresponds a discrete society $\Sigma^{n}$ obtained by taking the 'projection' of $\Sigma$ on $\Theta^{n}$. A strategy profile $S$ in the continuum society $\Sigma$ is called a limit equilibrium if the sequence $S^{n}$ of (unique) maximal subgame perfect equilibria in the discrete games $\Gamma\left(\Sigma^{n}\right)$ converges to $S$.

In order to formalize the idea of approximating a continuum society by a sequence of discrete societies, we need the following definitions. Let $\Sigma=\left(\Theta, u, \rho, \pi_{0}\right)$ be a continuum society where (a) $\Theta$ is a non-atomic separable metric space, (b) $u: \Theta \times \Theta \rightarrow \mathbb{R}$ is continuous and (c) $\rho$ and $\pi_{0}$ are strictly positive measures on $\Theta^{6}$.

\footnotetext{
${ }^{6} \mathrm{~A}$ measure on a metric space is called strictly positive if it assigns strictly positive mass to each non-empty, open subset.
} 
A discrete grid on $\Theta$ is a pair $(\hat{\Theta}, P)$ where $\hat{\Theta}=\left\{\theta_{1}, \theta_{2}, \ldots\right\}$ is a discrete subset of $\Theta$ and $P=\left\{P_{1}, P_{2}, \ldots\right\}$ is a partition of $\Theta$ such that $\theta_{k} \in P_{k}$ and $P_{k}$ contains a non-empty open subset for all $k$. The grid induces a discrete society $\hat{\Sigma}=\left(\hat{\Theta}, \hat{u}, \hat{\rho}, \hat{\pi}_{0}\right)$ where (a) the set of agents is $\hat{\Theta}$, (b) the utility profile $\hat{u}: \hat{\Theta} \times \hat{\Theta} \rightarrow \mathbb{R}$ is the restriction of $u$ on $\hat{\Theta} \times \hat{\Theta}$, (c) $\hat{\rho}$ is the measure on $\hat{\Theta}$ given by $\hat{\rho}\left(\theta_{k}\right)=\rho\left(P_{k}\right)$ for all $\theta_{k} \in \hat{\Theta}$ and (d) $\hat{\pi}_{0}$ is the measure on $\hat{\Theta}$ with $\hat{\pi}_{0}\left(\theta_{k}\right)=\pi_{0}\left(P_{k}\right)$ for all $\theta_{k} \in \hat{\Theta} .7$ The discrete society $\hat{\Sigma}$ is called the discrete projection of $\Sigma$ induced by the grid $(\hat{\Theta}, P)$. We write $\hat{\Sigma}=\Sigma(\hat{\Theta}, P)$.

For a given discrete grid $(\hat{\Theta}, P)$, let $\bar{u}: \Theta \times \Theta \rightarrow \mathbb{R}$ be the step function induced by $u$ and $(\hat{\Theta}, P)$, given by

$$
\bar{u}(\theta ; x)=u\left(\theta_{k} ; x_{l}\right)
$$

if $\theta \in P_{k}$ and $x \in P_{l}$. Hence, $\bar{u}$ is the unique function on $\Theta \times \Theta$ which coincides with $u$ on $\hat{\Theta} \times \hat{\Theta}$ and is constant on the induced partition elements of $\Theta \times \theta$.

Let $\Sigma$ be a continuum society and $\Sigma^{n}=\Sigma\left(\Theta^{n}, P^{n}\right)$ a sequence of dicrete projections with corresponding step functions $\bar{u}^{n}$. We say that $\Sigma^{n}$ converges to $\Sigma$ if (a) $\Theta^{n} \subset \Theta^{n+1}$ for all $n$, (b) $\cup_{n} \Theta^{n}$ is dense in $\Theta$ and (c) $\bar{u}^{n}$ converges uniformly to $u .^{8}$

Let $\Sigma^{n}$ be a sequence of discrete projections converging to the continuum society $\Sigma$ and let $S^{n}$ be the unique maximal subgame perfect equilibrium in $\Gamma\left(\Sigma^{n}\right)$. It remains to define the limit behavior in $\Sigma$ induced by the sequence $S^{n}$. A history independent ${ }^{9}$ strategy profile in the continuum society $\Sigma$ is a profile $S=\left(S_{t}(\theta)\right)_{t \leq T, \theta \in \Theta}$ where $S_{t}(\theta) \subset \Theta$ for all $t$ and $\theta$. The limit behavior induced by $S^{n}$ is defined as the history independent strategy profile $\lim S^{n}$ in $\Sigma$ given by

$$
\lim S_{t}^{n}(\theta)=\left\{x \in \Theta \mid \forall n \exists\left(\theta^{n}, x^{n}\right) \in \Theta^{n} \times \Theta^{n} \text { s.t. } x^{n} \in S_{t}^{n}\left(\theta^{n}\right) \text { and } \lim _{n \rightarrow \infty}\left(\theta^{n}, x^{n}\right)=(\theta, x)\right\}
$$

Intuitively, it means that $\theta$ supports $x$ in $\lim S^{n}$ if we can find representatives $\theta^{n}$ and $x^{n}$ arbitrarily close to $\theta$ and $x$ such that $x^{n}$ is supported by $\theta^{n}$ in some of the approximating equilibria.

Now, we have all the ingredients to define limit equilibria. A history independent strategy profile $S$ in the continuum society $\Sigma$ is called a limit equilibrium if there is a sequence $\Sigma^{n}$ of discrete projections converging to $\Sigma$ and a sequence $S^{n}$ of maximal subgame perfect equilibria in $\Gamma\left(\Sigma^{n}\right)$ such that $S$ is almost equal to $\lim S^{n}$.

Here, two strategies profiles $S$ and $\vec{S}$ in $\Sigma$ are said to be almost equal if for every $t$ and $\theta$ the difference between the sets $S_{t}(\theta)$ and $\bar{S}_{t}(\theta)$ has $\rho$-measure zero.

\footnotetext{
${ }^{7}$ The measures $\hat{\rho}$ and $\hat{\pi}_{0}$ are strictly positive since $\rho$ and $\pi_{0}$ are assumed to be strictly positive measures on $\Theta$ and all partition elements in $P$ contain non-empty open subsets.

${ }^{8}$ A sequence of functions $f_{n}: A \rightarrow \mathbb{R}$ is said to converge uniformly to $f: A \rightarrow \mathbb{R}$ if for every $\varepsilon>0$ there exists an $N \in \mathbb{N}$ such that $\left|f(x)-f_{n}(x)\right|<\varepsilon$ for all $n \geq N$ and all $x \in A$.

${ }^{9}$ We know, by Theorem 3.1, that the maximal subgame perfect equilibria $S^{n}$ are all history independent. Since we want to define the limit behavior induced by $S^{n}$ in $\Sigma$, it suffices to restrict attention to history independent strategy profiles in $\Sigma$.
} 


\subsection{Almost Uniqueness and Characterization}

In our theorem below, we prove the existence of a special limit equilibrium $S$ and show that every limit equilibrium is almost equal to $S$. The special limit equilibrium $S$ is characterized by value functions $V_{t}: \Theta \times \Theta \rightarrow \mathbb{R}$ given by the recursive formula

$$
V_{T}(\theta ; x)=\lambda_{T} u(\theta ; x)
$$

and

$$
V_{t}(\theta ; x)=\lambda_{t} u(\theta ; x)+V_{t+1}(\theta ; x)+\int_{y: V_{t+1}(x ; y) \geq 0} V_{t+1}(\theta ; y) d \rho(y)
$$

for all $t<T$. Hence, the value functions $V_{t}$ are the continuum analogues of the value functions in discrete societies.

In order to obtain our result, we impose two weak regularity conditions on the continuum society $\Sigma$ which are called the boundedness condition and the indifference condition. The society $\Sigma$ is said to satisfy the boundedness condition if there is a number $a<0$ and a number $M>0$ such that

$$
\rho\left(\left\{x \in \Theta \mid V_{t}(\theta ; x) \geq a\right\}\right) \leq M
$$

for all $t$ and $\theta$. We say that the game satisfies the indifference condition if

$$
\rho\left(\left\{x \in \Theta \mid V_{t}(\theta ; x)=0\right\}\right)=0
$$

is satisfied for all $t$ and $\theta$. ${ }^{10}$ In words, this means that the group of agents $x$ for which $\theta$ is indifferent always is neglectably small.

Theorem 4.1. Let $\Sigma$ be a continuum society satisfying the boundedness condition and the indifference condition. Let $S$ be the history independent strategy profile in $\Sigma$ given by

$$
S_{t}(\theta)=\left\{x \mid V_{t}(\theta ; x) \geq 0\right\}
$$

for all $t$ and $\theta$. Then, $S$ is a limit equilibrium and every limit equilibrium is almost equal to $S$.

Proof. Let $\Sigma^{n}$ be a sequence of discrete projections converging to $\Sigma$, induced by the discrete grids $\left(\Theta^{n}, P^{n}\right)$. Let $S^{n}$ be the corresponding sequence of maximal subgame perfect equilibria. We define the history independent strategy profile $\bar{S}$ in $\Sigma$ by $\bar{S}=\lim S^{n}$. We proceed in three steps. First, we prove that the corresponding sequence of value functions $V_{t}^{n}$ of the discrete approximating societies converges to the value function $V_{t}$ in $\Sigma$. Secondly, it is shown that $\bar{S}$ is almost equal to the strategy profile $S$ defined in the theorem, implying that $S$ is a limit equilibrium. Finally, we prove that every limit equilibrium is almost equal to $S$.

\footnotetext{
${ }^{10}$ This condition is typically satisfied if $\Theta$ is an Euclidean space, $\rho$ is measurable with respect to the Lebesgue measure and $u$ is such that $\{x \mid u(\theta ; x)=0\}$ is a countable set for all $\theta$. Since this is the standard framework we are thinking of, the indifference condition does hardly impose any restrictions.
} 
Step 1. Convergence of discrete value functions to continuum value functions.

Let $\Sigma^{n}$ be the discrete projection generated by the discrete grid $\left(\Theta^{n}, P^{n}\right)$. For every $n$ and every $t$ let $V_{t}^{n}: \Theta^{n} \times \Theta^{n} \rightarrow \mathbb{R}$ be the value function at stage $t$ in the discrete society $\Sigma^{n}$, as defined in the previous section. We define the step functions $\bar{V}_{t}^{n}: \Theta \times \Theta \rightarrow \mathbb{R}$ by

$$
\bar{V}_{t}^{n}(\theta ; x)=V_{t}^{n}\left(\theta_{k} ; x_{l}\right)
$$

where $\theta_{k}, x_{l} \in \Theta^{n}$ are in the same $P^{n}$-partition element as $\theta$ and $x$ respectively.

Lemma 4.2. For every $t$, the sequence of functions $\bar{V}_{t}^{n}$ converges uniformly to $V_{t}$.

Proof. We proceed by induction on $t$. For every $n$ let $\bar{u}^{n}: \Theta \times \Theta \rightarrow \mathbb{R}$ be the step function generated by $u$ and $\left(\Theta^{n}, P^{n}\right)$, as defined above. Since $\Sigma^{n}$ converges to $\Sigma$, the sequence $\bar{u}^{n}$ converges uniformly to $u$.

If $t=T$, then $V_{T}^{n}=\lambda_{T} u^{n}$ and hence $\bar{V}_{T}^{n}=\lambda_{T} \bar{u}^{n}$. Using the fact that $\bar{u}^{n}$ converges uniformly to $u$ and $V_{T}=\lambda_{T} u$ leads to the conclusion that $\bar{V}_{n}^{T}$ converges uniformly to $V_{T}$.

Now, let $t<T$. Let $n$ be fixed, $(\theta ; x)$ an arbitrary point in $\Theta \times \Theta$ and $\left(\theta_{k} ; x_{l}\right) \in \Theta^{n} \times \Theta^{n}$ such that $\theta$ and $x$ are in the same $P^{n}$-partition set as $\theta_{k}$ and $x_{l}$ respectively. Let $u^{n}$ be the utility profile and $\rho^{n}$ the support sensitivity measure in $\Sigma^{n}$. Then, by definition

$$
\begin{aligned}
\bar{V}_{t}^{n}(\theta ; x) & =V_{t}^{n}\left(\theta_{k} ; x_{l}\right) \\
& =\lambda_{t} u^{n}\left(\theta_{k} ; x_{l}\right)+V_{t+1}^{n}\left(\theta_{k} ; x_{l}\right)+\sum_{y_{n} \in \Theta^{n}: V_{t+1}^{n}\left(x_{l} ; y_{n}\right) \geq 0} V_{t+1}^{n}\left(\theta_{k} ; y_{n}\right) \cdot \rho^{n}\left(y_{n}\right) .
\end{aligned}
$$

Since $\rho^{n}$ is the 'contraction' of $\rho$ on $\Theta^{n}$ induced by $P^{n}$ and $\bar{V}_{t+1}^{n}$ is the step function on $\Theta \times \Theta$ which is constant on $P^{n}$-partition elements and coincides with $V_{t+1}^{n}$ on $\Theta^{n} \times \Theta^{n}$, it follows that

$$
\begin{aligned}
\bar{V}_{t}^{n}(\theta ; x) & =\lambda_{t} u^{n}\left(\theta_{k} ; x_{l}\right)+V_{t+1}^{n}\left(\theta_{k} ; x_{l}\right)+\int_{y \in \Theta: \bar{V}_{t+1}^{n}(x ; y) \geq 0} \bar{V}_{t+1}^{n}(\theta ; y) d \rho(y) \\
& =\lambda_{t} \bar{u}^{n}(\theta ; x)+\bar{V}_{t+1}^{n}(\theta ; x)+\int_{y \in \Theta} \bar{V}_{t+1}^{n}(\theta ; y) s_{t+1}^{n}(x ; y) d \rho(y),
\end{aligned}
$$

where $s_{t+1}^{n}: \Theta \times \Theta \rightarrow\{0,1\}$ is the function defined by

$$
s_{t+1}^{n}(x ; y)= \begin{cases}1, & \text { if } \bar{V}_{t+1}^{n}(x ; y) \geq 0 \\ 0, & \text { otherwise. }\end{cases}
$$

In the last equation, we used the definitions of $\bar{u}^{n}$ and $\bar{V}_{t+1}^{n}$. Using the recursive formula for $V_{t}$ we have

$$
V_{t}(\theta ; x)=\lambda_{t} u(\theta ; x)+V_{t+1}(\theta ; x)+\int_{y \in \Theta} V_{t+1}(\theta ; y) s_{t+1}(x ; y) d \rho(y)
$$


where

$$
s_{t+1}(x ; y)= \begin{cases}1, & \text { if } V_{t+1}(x ; y) \geq 0 \\ 0, & \text { otherwise. }\end{cases}
$$

By combining equations (4.1) and (4.2) we obtain that

$$
\begin{aligned}
\left|\bar{V}_{t}^{n}(\theta ; x)-V_{t}(\theta ; x)\right| \leq & \lambda_{t}\left|\bar{u}^{n}(\theta ; x)-u(\theta ; x)\right|+\left|\bar{V}_{t+1}^{n}(\theta ; x)-V_{t+1}(\theta ; x)\right| \\
& +\int_{y}\left|\bar{V}_{t+1}^{n}(\theta ; y) s_{t+1}^{n}(x ; y)-V_{t+1}(\theta ; y) s_{t+1}(x ; y)\right| d \rho(y) .
\end{aligned}
$$

Let $\varepsilon>0$ be given. We show that there is an $N$ such that $\left|\bar{V}_{t}^{n}(\theta ; x)-V_{t}(\theta ; x)\right|<\varepsilon$ for all $n \geq N$ and all $(\theta ; x)$ in $\Theta \times \Theta$.

Let the three terms on the right hand side of (4.3) be denoted by $A^{n}(\theta ; x), B^{n}(\theta ; x)$ and $C^{n}(\theta ; x)$. Since $\bar{u}^{n}$ converges uniformly to $u$ we can find $N_{1} \in \mathbb{N}$ such that $A^{n}(\theta ; x)<\frac{\varepsilon}{3}$ for all $n \geq N_{1}$ and all $(\theta ; x)$. Using the induction assumption that $\bar{V}_{t+1}^{n}$ converges uniformly to $V_{t+1}$ it follows that there is an $N_{2}$ such that $B^{n}(\theta ; x)<\frac{\varepsilon}{3}$ for all $n \geq N_{2}$ and all $(\theta ; x)$. It remains to show that we can find $N_{3}$ such that $C^{n}(\theta ; x)<\frac{\varepsilon}{3}$ for all $n \geq N_{3}$ and all $(\theta ; x)$.

By the boundedness condition, there is $a<0$ and an $M>0$ such that $\rho\left(\left\{x \mid V_{t+1}(\theta ; x) \geq\right.\right.$ $a\}) \leq M$ for all $\theta$. Choose $b>0$ such that $b<\min \left\{\frac{\varepsilon}{27 M},-a\right\}$. Then the term $C^{n}(\theta ; x)$ can be written as the sum

$$
\begin{aligned}
C^{n}(\theta ; x)= & \int_{y: V_{t+1}(x ; y) \leq-b}\left|\bar{V}_{t+1}^{n}(\theta ; y) s_{t+1}^{n}(x ; y)-V_{t+1}(\theta ; y) s_{t+1}(x ; y)\right| d \rho(y) \\
& +\int_{y:-b<V_{t+1}(x ; y)<b}\left|\bar{V}_{t+1}^{n}(\theta ; y) s_{t+1}^{n}(x ; y)-V_{t+1}(\theta ; y) s_{t+1}(x ; y)\right| d \rho(y) \\
& +\int_{y: V_{t+1}(x ; y) \geq b}\left|\bar{V}_{t+1}^{n}(\theta ; y) s_{t+1}^{n}(x ; y)-V_{t+1}(\theta ; y) s_{t+1}(x ; y)\right| d \rho(y) .
\end{aligned}
$$

Let the three terms on the right hand side be denoted by $C_{1}^{n}(\theta ; x), C_{2}^{n}(\theta ; x)$ and $C_{3}^{n}(\theta ; x)$ respectively. We show that for $i=1,2,3$, the term $C_{i}^{n}(\theta ; x)<\frac{\varepsilon}{9}$ for all $(\theta ; x)$ if $n$ is large enough.

We start with $C_{1}^{n}(\theta ; x)$. Since $\bar{V}_{t+1}^{n}$ converges uniformly to $V_{t+1}$, there exists $N_{3}$ such that $\left|\bar{V}_{t+1}^{n}(x ; y)-V_{t+1}(x ; y)\right|<\frac{b}{2}$ for all $n \geq N_{3}$ and all $(x ; y)$. Since we integrate only over $y$ 's with $V_{t+1}(x ; y) \leq-b$, it follows that $\bar{V}_{t+1}^{n}(x ; y)<0$ for all $y$ 's over which we integrate. This implies that $s_{t+1}^{n}(x ; y)=s_{t+1}(x ; y)=0$ for all $y$ in the integral and hence $C_{1}^{n}(\theta ; x)=0$ for all $n \geq N_{3}$ and all $(\theta ; x)$.

The term $C_{2}^{n}(\theta ; x)$ is bounded by

$$
\begin{aligned}
C_{2}^{n}(\theta ; x) \leq & \int_{y:-b<V_{t+1}(x ; y)<b}\left|\bar{V}_{t+1}^{n}(\theta ; y) s_{t+1}^{n}(x ; y)\right| d \rho(y) \\
& +\int_{y:-b<V_{t+1}(x ; y)<b}\left|V_{t+1}(\theta ; y) s_{t+1}(x ; y)\right| d \rho(y) .
\end{aligned}
$$


Since $\bar{V}_{t+1}^{n}$ converges uniformly to $V_{t+1}$ we can find $N_{4}$ such that $\left|\bar{V}_{t+1}^{n}(\theta ; y)-V_{t+1}(\theta ; y)\right|<b$ for all $n \geq N_{4}$ and all $(\theta ; y)$. Then, $\left|\bar{V}_{t+1}^{n}(\theta ; y)\right|<2 b$ for all $y$ over which we integrate and hence, for all $n \geq N_{4}$ and all $(\theta ; x)$,

$$
\begin{aligned}
C_{2}^{n}(\theta ; x) & \leq \int_{y:-b<V_{t+1}(x ; y)<b} 2 b d \rho(y)+\int_{y:-b<V_{t+1}(x ; y)<b} b d \rho(y) \\
& =3 b \cdot \rho\left(\left\{y \mid-b<V_{t+1}(x ; y)<b\right\}\right) .
\end{aligned}
$$

Since, by assumption, $b<-a$ (we refer to the constant $a<0$ of the boundedness condition), we have that $-b>a$ and therefore

$$
\left\{y \mid-b<V_{t+1}(x ; y)<b\right\} \subset\left\{y \mid V_{t+1}(x ; y) \geq a\right\}
$$

and hence, for all $n \geq N_{4}$ and all $(\theta ; x)$,

$$
C_{2}^{n}(\theta ; x) \leq 3 b \cdot \rho\left(\left\{y \mid V_{t+1}(x ; y) \geq a\right\}\right) \leq 3 b \cdot M
$$

by the boundedness condition. Since, by assumption, $b<\frac{\varepsilon}{27 M}$ it follows that $C_{2}^{n}(\theta ; x)<\frac{\varepsilon}{9}$ for all $n \geq N_{4}$ and all $(\theta ; x)$.

The term $C_{3}^{n}(\theta ; x)$ is given by

$$
C_{3}^{n}(\theta ; x)=\int_{y: V_{t+1}(x ; y) \geq b}\left|\bar{V}_{t+1}^{n}(\theta ; y) s_{t+1}^{n}(x ; y)-V_{t+1}(\theta ; y) s_{t+1}(x ; y)\right| d \rho(y) .
$$

Let $c>0$ be such that $c<\min \left\{\frac{b}{2}, \frac{\varepsilon}{9 M}\right\}$. Since $\bar{V}_{t+1}^{n}$ converges uniformly to $V_{t+1}$, we can find $N_{5}$ such that $\left|\bar{V}_{t+1}^{n}(x ; y)-V_{t+1}(x ; y)\right|<c$ for all $n \geq N_{5}$ and all $(x ; y)$. Then, since $c \leq \frac{b}{2}$, $V_{n}^{t+1}(x ; y)>0$ for all $y$ over which we integrate if $n \geq N_{5}$. Hence, for all $n \geq N_{5}, s_{t+1}^{n}(x ; y)=$ $s_{t+1}(x ; y)=1$ for all $y$ over which we integrate. Therefore, for all $n \geq N_{5}$ and all $(\theta ; x)$,

$$
\begin{aligned}
C_{3}^{n}(\theta ; x) & =\int_{y: V_{t+1}(x ; y) \geq b}\left|\bar{V}_{t+1}^{n}(\theta ; y)-V_{t+1}(\theta ; y)\right| d \rho(y) \\
& \leq \int_{y: V_{t+1}(x ; y) \geq b} c d \rho(y)=c \cdot \rho\left(\left\{y \mid V_{t+1}(x ; y) \geq b\right\}\right. \\
& \leq c \cdot \rho\left(\left\{y \mid V_{t+1}(x ; y) \geq a\right\}\right) \leq c \cdot M<\frac{\varepsilon}{9},
\end{aligned}
$$

since $c<\frac{\varepsilon}{9 M}$.

Now, let $N=\max \left\{N_{1}, \ldots, N_{5}\right\}$. Then, for all $n \geq N$ and all $(\theta ; x)$ we have that

$$
\left|\bar{V}_{t}^{n}(\theta ; x)-V_{t}(\theta ; x)\right| \leq A^{n}(\theta ; x)+B^{n}(\theta ; x)+C_{1}^{n}(\theta ; x)+C_{2}^{n}(\theta ; x)+C_{3}^{n}(\theta ; x)<\varepsilon .
$$

This completes the proof of the lemma. $\triangleleft$

This lemma implies that the value functions $V_{t}^{n}: \Theta^{n} \times \Theta^{n} \rightarrow \mathbb{R}$ converge pointwise to $V_{t}: \Theta \times \Theta \rightarrow \mathbb{R}$. Stated formally, we obtain the following result. 
Lemma 4.3. If the sequence $\left(\theta^{n} ; x^{n}\right) \in \Theta^{n} \times \Theta^{n}$ is such that $\lim _{n \rightarrow \infty}\left(\theta^{n} ; x^{n}\right)=(\theta ; x)$, then $\lim _{n \rightarrow \infty} V_{t}^{n}\left(\theta^{n} ; x^{n}\right)=V_{t}(\theta ; x)$.

Proof. For all $n$ we have that

$$
\begin{aligned}
\left|V_{t}^{n}\left(\theta^{n} ; x^{n}\right)-V_{t}(\theta ; x)\right| & =\left|\bar{V}_{t}^{n}\left(\theta^{n} ; x^{n}\right)-V_{t}(\theta ; x)\right| \\
& \leq\left|\bar{V}_{t}^{n}\left(\theta^{n} ; x^{n}\right)-V_{t}\left(\theta^{n} ; x^{n}\right)\right|+\left|V_{t}\left(\theta^{n} ; x^{n}\right)-V_{t}(\theta ; x)\right| .
\end{aligned}
$$

Let $\varepsilon>0$ be given. Since $V_{t}^{n}$ converges uniformly to $V_{t}$ and $V_{t}$ is continuous, there is $N$ such that $\left|\bar{V}_{t}^{n}\left(\theta^{n} ; x^{n}\right)-V_{t}\left(\theta^{n} ; x^{n}\right)\right|<\frac{\varepsilon}{2}$ and $\left|V_{t}\left(\theta^{n} ; x^{n}\right)-V_{t}(\theta ; x)\right|<\frac{\varepsilon}{2}$ for all $n \geq N$. Hence, $\left|V_{t}^{n}\left(\theta^{n} ; x^{n}\right)-V_{t}(\theta ; x)\right|<\varepsilon$ for all $n \geq N$ which implies that $\lim _{n \rightarrow \infty} V_{t}^{n}\left(\theta^{n} ; x^{n}\right)=V_{t}(\theta ; x) . \triangleleft$

Step 2. $S$ is a limit equilibrium.

By Theorem 3.1, we know that $\Gamma\left(\Sigma^{n}\right)$ has a unique maximal subgame perfect equilibrium $S^{n}$ characterized by the value functions $V_{t}^{n}$. Hence, by definition, $\bar{S}=\lim S^{n}$, where $S^{n}$ is given by

$$
S_{t}^{n}\left(\theta^{n}\right)=\left\{x^{n} \in \Theta^{n} \mid V_{t}^{n}\left(\theta^{n} ; x^{n}\right) \geq 0\right\}
$$

for all $t$ and all $\theta^{n} \in \Theta^{n}$. Now, we show that $\bar{S}$ is almost equal to the strategy profile $S$ in $\Sigma$ given by

$$
S_{t}(\theta)=\left\{x \in \Theta \mid V_{t}(\theta ; x) \geq 0\right\}
$$

for all $t$ and all $\theta \in \Theta$.

Let $t$ and $\theta \in \Theta$ be given. First, we prove that $\bar{S}_{t}(\theta) \subset S_{t}(\theta)$. Let $x \in \bar{S}_{t}(\theta)$. Since $\bar{S}=\lim S^{n}$, there is a sequence $\left(\theta^{n} ; x^{n}\right) \in \Theta^{n} \times \Theta^{n}$ converging to $(\theta ; x)$ such that $x^{n} \in S_{t}^{n}\left(\theta^{n}\right)$ for all $n$. Hence, $V_{t}^{n}\left(\theta^{n} ; x^{n}\right) \geq 0$ for all $n$. Since $V_{t}^{n}$ converges pointwise to $V_{t}$ it follows that $V_{t}(\theta ; x) \geq 0$ and hence $x \in S_{t}(\theta)$.

Now, we show that $S_{t}(\theta) \backslash \bar{S}_{t}(\theta)$ has $\rho$-measure zero. Let $x \in S_{t}(\theta)$, hence $V_{t}(\theta ; x) \geq 0$.

Suppose that $V_{t}(\theta ; x)>0$. Then, since $V_{t}^{n}$ converges pointwise to $V_{t}$, there is a sequence $\left(\theta^{n} ; x^{n}\right) \in \Theta^{n} \times \Theta^{n}$ converging to $(\theta ; x)$ such that $V_{t}^{n}\left(\theta^{n} ; x^{n}\right)>0$ for all $n$. Hence, $x^{n} \in S_{t}^{n}\left(\theta^{n}\right)$ for all $n$ which implies that $x \in \lim S^{n}=\bar{S}$. Therefore, $S_{t}(\theta) \backslash \bar{S}_{t}(\theta) \subset\left\{x \in \Theta \mid V_{t}(\theta ; x)=0\right\}$. But, by the indifference condition, $\rho\left(\left\{x \in \Theta \mid V_{t}(\theta ; x)=0\right\}\right)=0$, which implies that $S_{t}(\theta) \backslash \bar{S}_{t}(\theta)$ has $\rho$-measure zero. Given this fact, we may conclude that $\bar{S}_{t}(\theta)$ is almost equal to $S_{t}(\theta)$ for all $t$ and all $\theta$. This completes the proof of the fact that $S$ is a limit equilibrium.

Step 3. Every limit equilibrium is almost equal to $S$.

Let $\bar{S}$ be an arbitrary limit equilibrium. Then, by definition, there is a sequence $\Sigma^{n}$.of discrete projections converging to $\Sigma$ and a sequence $S^{n}$ of corresponding maximal subgame perfect equilibria such that $\bar{S}$ is almost equal to $\lim S^{n}$. In part (a) of the proof, we have shown that the special limit equilibrium $S$ is almost equal to $\lim S^{n}$, irrespective of the approximating sequence $\Sigma^{n}$ we choose. As a consequence, $\bar{S}$ is almost equal to $S$, which completes the proof of the theorem. 


\section{Single Peaked Preferences on the Real Line}

In this section, we apply the characterization of optimal support behavior in continuum societies, as presented in the previous section. Although we provide examples of continuum models, the reader should bear in mind that the qualitative features stressed in the examples will be shared by models of finite but large enough societies along the sequences converging to the limiting examples.

We focus on a group of agents which is represented by the real line. ${ }^{11} \mathrm{~A}$ possible interpretation is that agents are ordered according to some parameter which can be viewed as the agent's type. Agents which are close to each other on the line are similar whereas agents separated by a large distance are very different. In many situations, agents prefer types similar to their own. This can be modeled by single peaked preferences: for each agent $\theta$, the individual utility function $u(\theta ; \cdot): \mathbb{R} \rightarrow \mathbb{R}$ has a maximum at $\theta$ and utility decreases if the agent's type is further away from $\theta$.

As will become clear in the sections to come, the agents' support behavior depends crucially on the shape of the individual utility curves. It is important to know how utility changes if we move further away from the most preferred type. One possibility is that utility decreases faster if we are further away from the peak, meaning that individual utility functions are concave. This reflects situations where agents have strong preferences for similar types and strong 'objections' against very different types. This section is devoted to this particular class of preferences. We show that, under some symmetry conditions defined below, concave preferences induce support behavior in which agents always support subsets of their friends. Recall that an agent $x$ is called $\theta$ 's friend if $u(\theta ; x) \geq 0$. Agents with concave preferences have therefore no incentives to support members with negative utility since this would lead to a decrease of their future utility. Before deriving the result, we specify the setting and introduce the symmetry assumptions.

Consider the continuum society $\Theta=\mathbb{R}$. We assume that $\rho$ is the Lebesgue-measure on $\mathbb{R}$. Instead of $d \rho(y)$ we write $d y$. We say that the utility profile $u: \Theta \times \Theta \rightarrow \mathbb{R}$ is strictly concave if the individual utility functions $u(\theta ; \cdot): \mathbb{R} \rightarrow \mathbb{R}$ are strictly concave for all $\theta$. The utility profile $u$ is called anonymous if $u(\theta ; x)=u(\theta+a ; x+a)$ for all $\theta, x, a$. In words, this means that every agent has the same utility function, up to translation. We say that $u$ is diagonally symmetric if $u(\theta ; \theta+a)=u(\theta ; \theta-a)$ for all $\theta, a$. Note that strict concavity and diagonal symmetry imply that $u(\theta ; \cdot)$ has a single peak at $\theta$.

Theorem 5.1. Let $u$ be strictly concave, anonymous and diagonally symmetric and let $\lambda_{T}>0$. Then, the limit equilibrium $S$ of Theorem 4.1 is the almost unique limit equilibrium and $S$ is such that agents will always support a subset of their friends. If, moreover, $\lambda_{t}=0$ for all $t<T$, then the group $S_{t}(\theta)$ of agents supported by $\theta$ increases monotonically over time for every $\theta$.

Our conjecture is that monotonicity of support also holds if $\lambda_{t}>0$ for $t<T$. However, we did not manage to find a proof yet.

\footnotetext{
${ }^{11}$ We choose the real line instead of a bounded interval for the sake of symmetry. Bounded intervals have the technical disadvantage that boundary effects have to be taken into account. Our results would (approximately) hold for agents in a bounded interval located far from the boundaries and can therefore be interpreted as local results.
} 
The proof of this theorem can be found in the appendix.

The theorem above states that agents with concave, anonymous and diagonally symmetric preferences have no incentives to support enemies. The recursive formula for the value functions $V_{t}$ has been applied to derive this qualitative result. In the remainder of this section, we exploit this recursive formula to explicitly compute the support behavior for two special classes of concave preferences, namely quadratic and exponential preferences. It turns out that in both cases the group supported by a particular agent increases monotonically over time: a result which strengthens our conjecture stated directly after the theorem. Moreover, the group supported by $\theta$ increases faster as time evolves.

Next, we turn to normal preferences as an example of non-concave preferences. In contrast to the previous results, now agents will support enemies at all but the last stage and the group supported by an agent decreases monotonically over time.

Throughout the remainder of this section, we assume that $\Theta$ is the real line, $\lambda_{t}=1$ for all $t$ and $\rho$ is the Lebesgue measure.

\subsection{Quadratic Preferences}

Assume that the utility profile $u$ is given by

$$
u(\theta ; x)=-(x-(\theta-1))(x-(\theta+1)) .
$$

Hence, $u(\theta ; \cdot)$ has a maximum at $\theta$ and roots at $\theta-1$ and $\theta+1$. Obviously, $u$ is strictly concave, anonymous and diagonally symmetric and Theorem 5.1 assures that there is an almost unique limit equilibrium $S$ in which agents will never support enemies. Using the recursive formula for the value functions, we are able to prove that all value functions are quadratic functions of the form

$$
V_{t}(\theta ; x)=-a_{t}\left(x-\left(\theta-b_{t}\right)\right)\left(x-\left(\theta+b_{t}\right)\right)
$$

where the coefficients $a_{t}, b_{t}$ are given by the recursive equation

$$
\begin{aligned}
a_{T} & =1, b_{T}=1 \\
a_{t} & =2 a_{t+1} b_{t+1}+1+a_{t+1} \\
b_{t} & =\sqrt{\frac{4 a^{t+1}\left(b_{t+1}\right)^{3}+3+3 a^{t+1}\left(b_{t+1}\right)^{2}}{6 a^{t+1} b_{t+1}+3+3 a^{t+1}}} .
\end{aligned}
$$

Here, the coefficients $a_{t}$ and $b_{t}$ do not depend on $\theta$.

In the limit equilibrium $S$ agent $\theta$ supports the interval $\left[\theta-b_{t}, \theta+b_{t}\right]$ at stage $t$. Hence, determining the limit equilibrium $S$ is simply a matter of computing the parameters $b_{t}$ given by the recursive formula (5.1). Exploiting the formula, it can be shown that $b_{t}$ is strictly increasing over time and hence the group of agents supported by $\theta$ monotonically increases over time for all $\theta$. If the number of stages $T$ is large and $t$ is small,

$$
b_{t} \approx b_{t+1} \sqrt{\frac{4 b^{t+1}+3}{6 b^{t+1}+3}}
$$


The last property follows from formula (5.1) and the observation that $a_{t}$ tends to infinity if $T-t$ is very large. It implies that $b_{t}$ tends to zero if $T-t$ is very large. Intuitively, this means that if many stages are involved, agents support only very small groups at the beginning. The following picture shows the evolution of $b_{t}$ in a game with 15 stages.

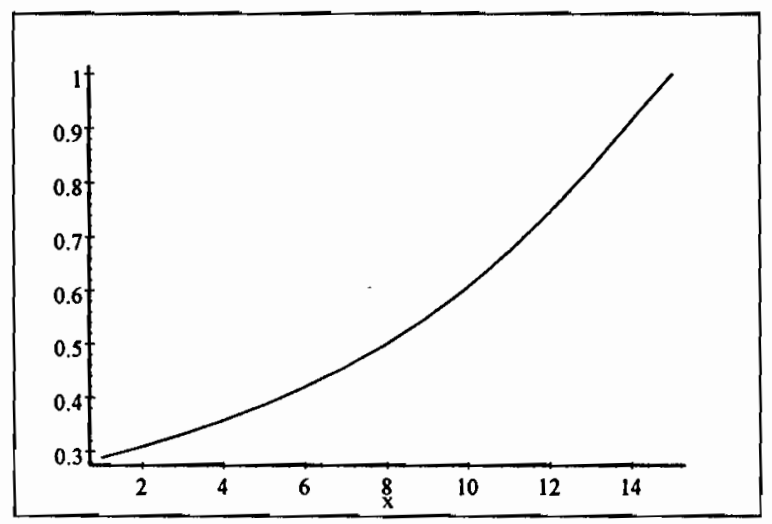

Figure 1

Evolution of support with quadratic preferences

\subsection{Exponential Preferences}

Assume that the utility profile $u$ has the form

$$
u(\theta ; x)=e+\frac{1}{e}-e^{x-\theta}-e^{\theta-x} .
$$

The function $u(\theta ; \cdot)$ has a maximum at $\theta$ and roots at $\theta-1$ and $\theta+1$. Again, $u$ is concave, anonymous and diagonally symmetric and hence Theorem 5.1 applies. Using the recursive formula for the value functions, it can be shown that all value functions are exponential functions of the form

$$
V_{t}(\theta ; x)=a_{t}-b_{t}\left(e^{x-\theta}+e^{\theta-x}\right)
$$

where $a_{t}$ and $b_{t}$ are parameters which do not depend on $\theta$. The limit equilibrium $S$ is such that at every stage $t$ agent $\theta$ supports $S_{t}(\theta)=\left[\theta-c_{t}, \theta+c_{t}\right]$ with $c_{t}$ being independent of $\theta$. The numbers $a_{t}, b_{t}$ and $c_{t}$ are given by the recursive equation

$$
\begin{aligned}
a_{T} & =e+\frac{1}{e}, b_{T}=1, c_{T}=1, \\
a_{t} & =e+\frac{1}{e}+a_{t+1}+2 a_{t+1} c_{t+1}, \\
b_{t} & =1+b_{t+1}+b_{t+1}\left(e^{c_{t+1}}-e^{-c_{t+1}}\right), \\
c_{t} & =\operatorname{arccosh}\left(\frac{a_{t}}{2 b^{t}}\right) .
\end{aligned}
$$

Computing the limit equilibrium $S$ is therefore equivalent to calculating the parameters $c_{t}$ given by the recursive formula above. The following picture shows the evolution of $c_{t}$ over time in a game with 15 stages. 


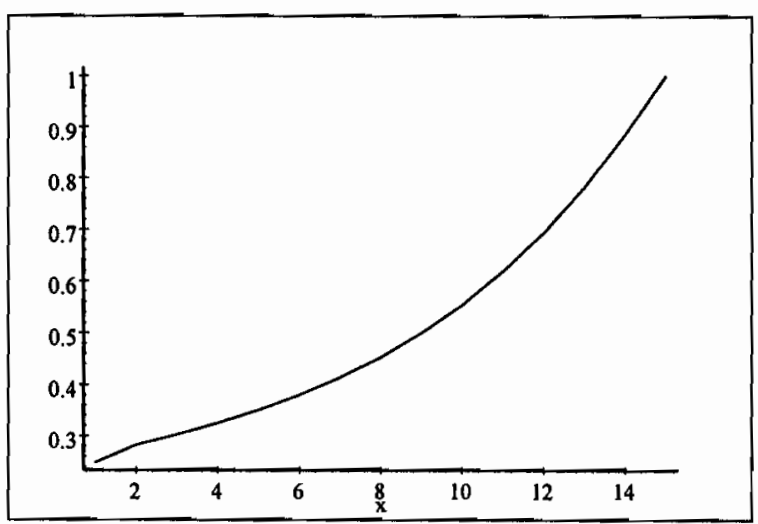

Figure 2

Evolution of support with exponential preferences

Similar to the case with quadratic preferences, the group supported by agent $\theta$ strictly increases over time and the rate of increase grows as time evolves.

\subsection{Normal Preferences}

Assume that the utility profile $u$ is given by

$$
u(\theta ; x)=e^{-(x-\theta)^{2}}-\frac{1}{e} .
$$

The function $u(\theta ; \cdot)$ has a maximum at $\theta$ and roots at $\theta-1$ and $\theta+1$. Hence, $u$ is diagonally symmetric and anonymous but not concave. Unfortunately, the value functions $V_{t}$ can not be written in the form

$$
V_{t}(\theta ; x)=a e^{-(x-\theta)^{2}}-b
$$

and can therefore not be parametrized as has been the case with quadratic and exponential preferences. This complicates our attempt to undertake simulations with many stages, since the computer package has to calculate the complete value function at each stage. A simulation with four stages shows that the curves of the individual value functions $V_{t}(\theta ; \cdot)$ are very close to normal. The pictures below show the curves of $V_{t}(0 ; \cdot)$ at stages 2,3 and 4 respectively. Since $u$ is diagonally symmetric and anonymous, the proof of Theorem 5.1 assures that the value function $V_{t}$ is anonymous and therefore every individual value function $V_{t}(\theta ; \cdot)$ is equal to $V_{t}(0 ; \cdot)$ after translation.

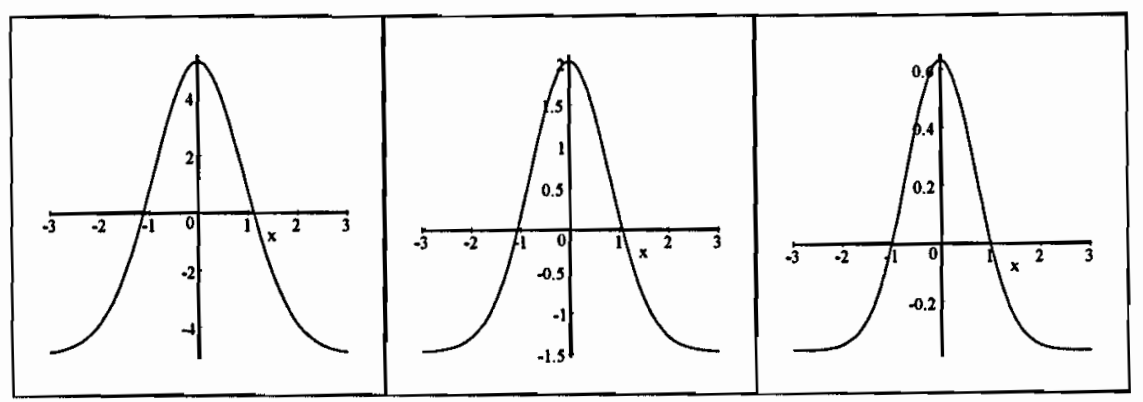




\section{Figure 3}

Evolution of value function with normal preferences

Since the boundedness condition and the indifference condition are clearly satisfied, the value functions characterize an almost unique limit equilibrium $S$. As can be seen in the picture, the group supported by agent $\theta$ in $S$ contains enemies at all but the last stage and decreases over time. More exactly, the limit equilibrium is such that agent $\theta$ at stage $t$ supports the set $S_{t}(\theta)=$ $\left[\theta-a_{t}, \theta+a_{t}\right]$ where $a_{t}$ does not depend on $\theta$ and is given by the following table:

\begin{tabular}{|l|l|l|l|l|}
\hline$t$ & 1 & 2 & 3 & 4 \\
\hline$a_{t}$ & 1.15 & 1.11 & 1.06 & 1.00 \\
\hline
\end{tabular}

\section{Stationary Support}

In Section 5 we have seen that quadratic and exponential preferences lead to increasing support over time, whereas normal preferences induce decreasing support as time evolves. These results raise the question whether we can find circumstances under which support remains constant over time. In this section we provide a sufficient condition for stationary support behavior and illustrate the result with an example.

A utility profile $u$ in a continuum society is said to induce stationary support if the limit equilibrium $S$ (by which we mean the limit equilibrium in Theorem 4.1) is such that for every $\theta$ the set $S_{t}(\theta)$ remains constant over time. In the theorem we present a sufficient condition on $u$ to induce stationary support. To this purpose, we define the operator $A$ transforming a function $f: \Theta \times \Theta \rightarrow \mathbb{R}$ into the function $A f: \Theta \times \Theta \rightarrow \mathbb{R}$ given by

$$
A f(\theta ; x)=\int_{y: f(x ; y) \geq 0} f(\theta ; y) d \rho(y) .
$$

Theorem 6.1. Let $\lambda_{t}>0$ for all $t$ and let the utility profile $u$ be such that $A u=\alpha u$ for some constant $\alpha>0$. Then, $u$ induces stationary support.

Proof. Let $u$ be such that $A u=\alpha u$ for some constant $\alpha>0$. Let $S$ be the limit equilibrium and $V_{t}$ the corresponding value functions. By definition, we have $V_{T}=\lambda_{T} u$ and $V_{t}=\lambda_{t} u+$ $V_{t+1}+A V_{t+1}$ for all $t<T$. In particular,

$$
\begin{aligned}
V_{T-1} & =\lambda_{T-1} u+V_{T}+A V_{T}=\lambda_{T-1} u+\lambda_{T} u+A \lambda_{T} u \\
& =\lambda_{T-1} u+\lambda_{T} u+\lambda_{T} A u=\left(\lambda_{T-1}+\lambda_{T}+\lambda_{T} \alpha\right) u
\end{aligned}
$$

By induction, it follows that $V_{t}$ is always a strictly positive multiple of $u$. Consequently, $S_{t}(\theta)=$ $\left\{x \mid V_{t}(\theta ; x) \geq 0\right\}=\{x \mid u(\theta ; x) \geq 0\}$ for all $t$ which means that $u$ induces stationary support. 
Example 6.1. Let $\Theta$ be the circle with unit length. For the sake of convenience, we represent $\Theta$ by the interval $[0,1]$. Let $\lambda_{t}>0$ for all $t$ and $\rho$ be the Lebesgue-measure on $[0,1]$. Let the utility profile $u$ be given by

$$
u(\theta ; x)=\cos (2 \pi(x-\theta))
$$

for all $\theta, x \in \Theta$. Hence, $u(\theta ; \cdot)$ has a maximum at $\theta$ and decreases as we go further away from $\theta$ on the circle. We show that $u$ induces stationary support.

For every $x \in \mathbb{R}$ let $[x]$ be the unique number in $[0,1)$ such that $x-[x] \in \mathbb{Z}$. Then $\{y \mid u(x ; y) \geq$ $0\}=\left\{[y] \mid y \in\left[x-\frac{1}{4}, x+\frac{1}{4}\right]\right\}$ and

$$
\begin{aligned}
A u(\theta ; x) & =\int_{x-\frac{1}{4}}^{x+\frac{1}{4}} u(\theta ;[y]) d y=\int_{x-\frac{1}{4}}^{x+\frac{1}{4}} \cos (2 \pi((y]-\theta)) d y=\int_{x-\frac{1}{4}}^{x+\frac{1}{4}} \cos (2 \pi(y-\theta)) d y \\
& =\frac{1}{2 \pi} \sin \left(2 \pi\left(x+\frac{1}{4}-\theta\right)\right)-\frac{1}{2 \pi} \sin \left(2 \pi\left(x-\frac{1}{4}-\theta\right)\right) \\
& =\frac{1}{\pi} \cos (2 \pi(x-\theta))=\frac{1}{\pi} u(\theta ; x) .
\end{aligned}
$$

Hence, $A u=\frac{1}{\pi} u$ and Theorem 6.1 assures that $u$ induces statonary support.

In the case where $\Theta$ is the real line, we did not yet manage to find an interesting utility profile $u$ which induces stationary support. Trivial but unappealing utility profiles that induce stationary support are, for instance, those in which each agent $\theta$ dislikes all other agents (i.e. $u(\theta ; x)<0$ for all $x)$. In this case, $\theta$ will always support nobody. An open question is still whether there exists a utility profile $u: \mathbb{R} \times \mathbb{R} \rightarrow \mathbb{R}$ inducing stationary support and satisfying the following properties: (a) $u$ is anonymous and diagonally symmetric, (b) $u(\theta ; \cdot)$ is positive at $[\theta-a, \theta+a]$ and negative outside for some $a>0$.

\section{Infinite Horizon}

Up to this point, we assumed that agents face a finite horizon, which is commonly known to all. In some cases, however, this assumption may not be appropriate. It is therefore worthwhile analyzing the case of an infinite (or uncertain) horizon. As will be shown in this section, the introduction of an infinite horizon induces a remarkable change in optimal support behavior: a phenomenon which is not surprising, given the important difference between a finite and an infinite horizon in the literature on repeated games. In general, uniqueness of subgame perfect equilibrium is lost when facing an infinite horizon and history dependent equilibria may arise. Before turning to history dependent equilibria, we first.analyze the class of history independent equilibria.

\subsection{History Independent Equilibria}

It turns out that, even when concentrating on history independent equilibria, the situation drastically changes when switching to an infinite horizon. As we will see, multiple history 
independent equilibria, and even multiple stationary equilibria may exist. Similar to the finite horizon case, they can be characterized by value functions.

In the sequel, we solely concentrate on discrete societies but similar arguments would hold when considering continuum societies. First, we try to characterize maximal subgame perfect equilibria with infinite horizon by using value functions. Consider a history independent maximal subgame perfect equilibrium $S$ for the finite horizon case, so $S=\left(S_{t}(\theta)\right)_{t \leq T, \theta \in \Theta}$. In the proof of Theorem 3.1, we have seen that the value functions $V_{t}$ are given by

$$
V_{t}(\theta ; x)=\left[\lambda_{t} u+\sum_{\tau=t+1}^{T} \lambda_{\tau} \prod_{l=t+1}^{\tau}\left(I+\tilde{s}_{l}\right) u\right](\theta ; x)
$$

where the operator $\tilde{s}_{l}$ transforms a function $f: \Theta \times \Theta \rightarrow \mathbb{R}$ into a function $\tilde{s}_{l} f: \Theta \times \Theta \rightarrow \mathbb{R}$ given by

$$
\tilde{s}_{l} f(\theta ; x)=\sum_{y \in S_{l}(x)} f(\theta ; y) \rho(y) .
$$

Now, let $S$ be a history independent strategy profile for the infinite horizon case, so $S=$ $\left(S_{t}(\theta)\right)_{t \in \mathbb{N}, \theta \in \Theta}$. In the same way as above, we may define the value functions $V_{t}, t \in \mathbb{N}$, induced by $S$ by

$$
V_{t}(\theta ; x)=\left[\lambda_{t} u+\sum_{\tau=t+1}^{\infty} \lambda_{\tau} \prod_{l=t+1}^{\tau}\left(I+\tilde{s}_{l}\right) u\right](\theta ; x)
$$

given that this expression exists. By essentially copying the proof of Theorem 3.1 we obtain the following characterization of history independent equilibria with infinite horizon.

Theorem 7.1. Let $S$ be a history independent strategy profile for the infinite horizon case. Suppose that $S$ is such that the value functions $\left(V_{t}\right)_{t \in \mathbb{N}}$ induced by $S$ exist. Then, $S$ is a maximal subgame perfect equilibrium if and only if $S_{t}(\theta)=\left\{x \mid V_{t}(\theta ; x) \geq 0\right\}$ for all $t$.

A disadvantage, in comparison with the finite horizon case, is that the value functions can no longer be computed recursively by backward induction, since there is no longer a final stage. Determining the value functions and verifying the condition of the theorem above can therefore become a complicated matter.

The theorem can be used, however, to provide a sufficient condition for stationary equilibria, similar to the one presented in Theorem 6.1 for a finite horizon. Let $A$ be the operator transforming a utility profile $u: \Theta \times \Theta \rightarrow \mathbb{R}$ into a new function $A u: \Theta \times \Theta \rightarrow \mathbb{R}$ given by

$$
A u(\theta ; x)=\sum_{y: u(x ; y) \geq 0} u(\theta ; y) \rho(y)
$$

Theorem 7.2. Let $A u=\alpha u$ for some number $\alpha>0$. Then, the stationary strategy profile $S$ in which at every stage, after any history, agent $\theta$ supports $S(\theta)=\{x \mid u(\theta ; x) \geq 0\}$ is a maximal subgame perfect equilibrium. 
Proof. Suppose that $A u=\alpha u$ for some $\alpha>0$. Let the stationary strategy profile $S$ be given by $S_{t}(\theta)=\{x \mid u(\theta ; x) \geq 0\}$ for all $t, \theta$. Then, by construction, $\tilde{s}_{l} u=A u=\alpha u$ for all $l \in \mathbb{N}$ and the value functions induced by the stationary strategy profile $S$ are given by

$$
\begin{aligned}
V_{t}(\theta ; x) & =\left[\lambda_{t} u+\sum_{\tau=t+1}^{\infty} \lambda_{\tau} \prod_{l=t+1}^{\tau}\left(I+\tilde{s}_{l}\right) u\right](\theta ; x)=\left[\lambda_{t} u+\sum_{\tau=t+1}^{\infty} \lambda_{\tau} \prod_{l=t+1}^{\tau}(I+A) u\right](\theta ; x) \\
& =\left[\lambda_{t} u+\sum_{\tau=t+1}^{\infty} \lambda_{\tau} \prod_{l=t+1}^{\tau}(1+\alpha) u\right](\theta ; x)=\left[\lambda_{t}+\sum_{\tau=t+1}^{\infty} \lambda_{\tau}(1+\alpha)^{\tau-t}\right] u(\theta ; x),
\end{aligned}
$$

so $V_{t}$ is a strictly positive multiple of $u$ for all $t$. Hence, $S(\theta)=\{x \mid u(\theta ; x) \geq 0\}=\left\{x \mid V_{t}(\theta ; x) \geq\right.$ 0 \} for all $t$ and $\theta$. Theorem 7.1 assures that $S$ is a maximal subgame perfect equilibrium.

The condition of the theorem above, however, is not necessary for stationary equilibria. In the following example, we are able to find two stationary equilibria: one which satisfies the sufficient condition and one which does not. As a byproduct, the example shows that there may exist multiple history independent equilibria (even multiple stationary equilibria) if the horizon is infinite.

Example 7.1. Consider a group $\Theta=\{1,2\}$ of agents for which the utilities $u(\theta ; x)$ are given by the matrix

\begin{tabular}{|c|c|}
\hline 2 & -1 \\
\hline-1 & 2 \\
\hline
\end{tabular}

We assume that $\rho(\theta)=1$ for both $\theta$ and $\lambda_{t}=\delta^{t}$ with $\delta=0.3$. Here, the discount factor $\delta$ is small enough as to ensure the existence of the value functions for any strategy profile. Let $S$ be the stationary strategy profile in which 1 always supports $\{1\}$ and 2 always supports $\{2\}$ at any stage and after any history. Let $S^{\prime}$ be the stationary strategy profile in which both 1 and 2 always support $\{1,2\}$. We show that both $S$ and $S^{\prime}$ are maximal subgame perfect equilibria.

It can be checked immediately that $A u=u$, so Theorem 7.2 implies that $S$ is a maximal subgame perfect equilibrium.

Now, let $\tilde{s}_{l}^{\prime} \equiv \tilde{s}^{\prime}$ be the operator at stage $l$ induced by $S^{\prime}$, as used in the definition of the value functions above. Then, the value function $V_{t}$ is given by

$$
V_{t}(\theta ; x)=\left[\lambda_{t} u+\sum_{\tau=t+1}^{\infty} \lambda_{\tau} \prod_{l=t+1}^{\tau}\left(I+\tilde{s}^{\prime}\right) u\right](\theta ; x)=\left[\lambda_{t} u+\sum_{\tau=t+1}^{\infty} \lambda_{\tau}\left(I+\tilde{s}^{\prime}\right)^{\tau-t} u\right](\theta ; x)
$$

By straightforward calculations, it can be shown that $V_{t}(\theta ; x)>0$ for all $(\theta ; x)$, and hence $S^{\prime}(\theta)=\{1,2\}=\left\{x \mid V_{t}(\theta ; x) \geq 0\right\}$ for all $t$ and $\theta$. Theorem 7.1 guarantees therefore that $S^{\prime}$ is a maximal subgame perfect equilibrium.

The intuition behind the equilibrium $S^{\prime}$ is the following: it is in agent $\theta$ 's interest to support the other agent $x$, since $x$ will support $\theta$ in all remaining stages. So, by supporting $x$, agent $\theta$ is implictly supporting himself. 
This equilibrium would break down if the horizon would be finite, since agents would only support themselves at the last stage, which makes it unattractive to support the other agent at the penultimate stage, which makes it unattractive to support the other agent at the stage before the penultimate stage and so on.

\subsection{History Dependent Equilibria}

If the agents face a finite horizon, the unique maximal subgame perfect equilibrium is history independent. By means of an example, we show that an infinite horizon may lead to history dependent equilibria.

Example 7.2. As in Example 7.1, consider a group $\Theta=\{1,2\}$ of agents in. which the utilities $u(\theta ; x)$ are given by

$$
\begin{array}{|c|c|}
\hline 2 & -1 \\
\hline-1 & 2 \\
\hline
\end{array}
$$

Again, $\rho(\theta)=1$ for both agents and $\lambda_{t}=\delta^{t}$ with $\delta=0.3$. Let $S$ be the history dependent strategy profile defined as follows:

Stage 1. Both agents support $\{1,2\}$.

Stage $t>1$. If at all previous stages, both agents supported $\{1,2\}$, then both agents continue to support $\{1,2\}$.

If at some previous stage, an agent did not support $\{1,2\}$, agent 1 supports $\{1\}$ and agent 2 supports $\{2\}$.

We prove that $S$ is a maximal subgame perfect equilibrium.

By symmetry of the game, it is sufficient to concentrate on agent 1's deviations. Suppose the game is at stage $t$ and both agents supported $\{1,2\}$ at all previous stages. Let the influence distribution at the beginning of stage $t$ be given by $\pi_{t-1}=\left(\pi_{t-1}(1), \pi_{t-1}(2)\right)$, where $\pi_{t-1}(\theta)$ denotes agent $\theta$ 's influence.

If agent 1 deviates at stage $t$, then agent 2 will support $\{2\}$ ever after. In Example 7.1 we have seen that both agents supporting only themselves is a maximal subgame perfect equilibrium (irrespective of the initial influence distribution). In particular, this means that if agent 2 supports $\{2\}$ ever after, the best for agent 1 to do is to support $\{1\}$ ever after. Using the recursive formula for the evolution of the influence distribution, as given in Section 2, it can be shown that the overall continuation utility for agent 1 by deviating at stage $t$, is less or equal to

$$
U_{1}^{\prime}=\frac{4 \delta^{t}}{1-2 \delta} \pi_{t-1}(1)+\left[\frac{2 \delta^{t}}{1-\delta}-\frac{2 \delta^{t}}{1-2 \delta}\right] \pi_{t-1}(2) .
$$

Assume on the other hand that agent 1 sticks to the strategy prescribed by $S$. Then, his overall continuation utility, starting at stage $t$, is equal to

$$
U_{1}=\left[\frac{3}{2} \frac{\delta^{t}}{1-\delta}+\frac{3}{2} \frac{\delta^{t}}{1-3 \delta}\right] \pi_{t-1}(1)+\left[-\frac{3}{2} \frac{\delta^{t}}{1-\delta}+\frac{3}{2} \frac{\delta^{t}}{1-3 \delta}\right] \pi_{t-1}(2) \text {. }
$$


It can be checked that $U_{1}^{\prime}<U_{1}$ for $\delta=0.3$ and for all $t$, so deviation is not beneficial.

Now, suppose that the game is at stage $t$ and some agent did not support $\{1,2\}$ at some of the previous stages. Then, $S$ prescribes that both agents should support only themselves in all remaining stages. In Example 7.1, we have seen that both agents supporting only themselves is a maximal subgame perfect equilibrium, which implies that deviation is not beneficial for agent 1 at this stage of the game. Hence, we may conclude that $S$ is a maximal subgame perfect equilibrium.

\section{Concluding Remarks}

Our main contributions in this paper are threefold. First, we propose a general game theoretical model to study situations in which agents pertaining to a society can transfer influence to other members by supporting them. We continue by showing that for discrete and continuum societies there is an essentially unique optimal support behavior if there is a finite horizon. Here, continuum societies should be seen as approximations of large discrete societies. Moreover, we present an easy to handle recursive formula which characterizes' the equilibrium. This formula is used to make qualitative statements on the support behavior of agents and to compute the equilibrium for specific utility classes.

The model presented can be extended in many different directions. One could, for instance, consider the situation in which agents can not support arbitrary groups of agents but face physical or financial restrictions. The model could also be extended to societies where the utilities $u(\theta ; x)$, reflecting the preferences for other society members, are not fixed but change over time. Or one could look at situations with continuous instead of discrete time. The techniques used to derive our results can probably be adapted to continuous time in the same way as related techniques in discrete dynamic programming have been adapted to continuous programming.

\section{Appendix}

Proof of Lemma 3.2. By definition of $\bar{f} \mu$ we have

$$
\bar{f} \mu(x)=\sum_{y} f(y ; x) \mu(y) \rho(x)
$$

and hence

$$
\begin{aligned}
\sum_{x} g(\theta ; x) \bar{f} \mu(x) & =\sum_{x} \sum_{y} g(\theta ; x) f(y ; x) \mu(y) \rho(x) \\
& =\sum_{x}\left[\sum_{y} g(\theta ; y) f(x ; y) \rho(y)\right] \mu(x) \\
& =\sum_{x} \tilde{f} g(\theta ; x) \mu(x)
\end{aligned}
$$


where the second equality is obtained by exchanging $x$ and $y . \triangleleft$

Proof of Lemma 3.3. By induction on $t-\tau$.

If $t=\tau$, then we have

$$
\begin{aligned}
U_{t}(\theta) & =\sum_{x} u(\theta ; x) \pi_{\tau}(x)=\sum_{x} u(\theta ; x)\left(\pi_{\tau-1}+\bar{s}_{\tau} \pi_{\tau-1}\right)(x) \\
& =\sum_{x} u(\theta ; x) \pi_{\tau-1}(x)+\sum_{x} u(\theta ; x) \bar{s}_{\tau} \pi_{\tau-1}(x) \\
& =\sum_{x} u(\theta ; x) \pi_{\tau-1}(x)+\sum_{x} \tilde{s}_{\tau} u(\theta ; x) \pi_{\tau-1}(x) \\
& =\sum_{x}\left(I+\tilde{s}_{\tau}\right) u(\theta ; x) \pi_{\tau-1}(\dot{x}),
\end{aligned}
$$

where the fourth equality follows from Lemma 3.2.

Now, let $t>\tau$ and let the statement be true for all $\tau^{\prime}<t^{\prime}$ with $t^{\prime}-\tau^{\prime}<t-\tau$. Then, in particular, the statement is true for $t$ and $\tau+1$ which means that

$$
\begin{aligned}
U_{t}(\theta) & =\sum_{x}\left[\prod_{l=\tau+1}^{t}\left(I+\tilde{s}_{l}\right) u\right](\theta ; x) \pi_{\tau}(x) \\
& =\sum_{x}\left[\prod_{l=\tau+1}^{t}\left(I+\tilde{s}_{l}\right) u\right](\theta ; x)\left(\pi_{\tau-1}+\bar{s}_{\tau} \pi_{\tau-1}\right)(x) \\
& =\sum_{x}\left[\prod_{l=\tau+1}^{t}\left(I+\tilde{s}_{l}\right) u\right](\theta ; x) \pi_{\tau-1}(x)+\sum_{x}\left[\prod_{l=\tau+1}^{t}\left(I+\tilde{s}_{l}\right) u\right](\theta ; x) \bar{s}_{\tau} \pi_{\tau-1}(x) \\
& =\sum_{x}\left[\prod_{l=\tau+1}^{t}\left(I+\tilde{s}_{l}\right) u\right](\theta ; x) \pi_{\tau-1}(x)+\sum_{x}\left[\tilde{s}_{\tau} \prod_{l=\tau+1}^{t}\left(I+\tilde{s}_{l}\right) u\right](\theta ; x) \pi_{\tau-1}(x) \\
& =\sum_{x}\left[\left(I+\tilde{s}_{\tau}\right) \prod_{l=\tau+1}^{t}\left(I+\tilde{s}_{l}\right) u\right](\theta ; x) \pi_{\tau-1}(x) \\
& =\sum_{x}\left[\prod_{l=\tau}^{t}\left(I+\tilde{s}_{l}\right) u\right](\theta ; x) \pi_{\tau-1}(x),
\end{aligned}
$$

where the fourth equality follows from Lemma 3.2. $\triangleleft$

Proof of Theorem 5.1. (a) First of all, we show that the value functions $V_{t}$ are strictly concave, anonymous and diagonally symmetric for all $t$. We prove the statement by induction on $t$.

If $t=T$ the statement is true since $V_{T}=\lambda_{T} u$ and $\lambda_{T}>0$. Now let $t<T$ and let the 
statement be true for all $\tau>t$. First we show anonimity of $V_{t}$. Since

$$
V_{t}(\theta ; x)=\lambda_{t} u(\theta ; x)+V_{t+1}(\theta ; x)+\int_{y: V_{t+1}(x ; y) \geq 0} V_{t+1}(\theta ; y) d y
$$

and $u, V_{t+1}$ are anonymous by assumption, it suffices to show that the function $F: \Theta \times \Theta \rightarrow \mathbb{R}$ given by

$$
F(\theta ; x)=\int_{y: V_{t+1}(x ; y) \geq 0} V_{t+1}(\theta ; y) d y
$$

is anonymous. By anonimity of $V_{t+1}$ it holds that

$$
\begin{aligned}
F(\theta+a ; x+a) & =\int_{y: V_{t+1}(x+a ; y) \geq 0} V_{t+1}(\theta+a ; y) d y=\int_{y: V_{t+1}(x ; y-a) \geq 0} V_{t+1}(\theta ; y-a) d y \\
& =\int_{z: V_{t+1}(x ; z) \geq 0} V_{t+1}(\theta ; z) d z=F(\theta ; x)
\end{aligned}
$$

where the third equation follows by substituting $z=y-a$. Hence, $F$ is anonymous.

Next, we show diagonal symmetry of $V_{t}$. Since by assumption $u$ and $V_{t+1}$ are diagonally symmetric it suffices to show that $F$ is diagonally symmetric. By definition, we have that

$$
F(\theta ; \theta+a)=\int_{y: V_{t+1}(\theta+a ; y) \geq 0} V_{t+1}(\theta ; y) d y .
$$

Since $V_{t+1}$ is diagonally symmetric we have

$$
V_{t+1}(\theta ; y)=V_{t+1}(\theta ; \theta+(y-\theta))=V_{t+1}(\theta ; \theta-(y-\theta))=V_{t+1}(\theta ; 2 \theta-y)
$$

for all $y$. By anonimity and diagonal symmetry of $V_{t+1}$ we have

$$
V_{t+1}(\theta+a ; y)=V_{t+1}(\theta-a ; y-2 a)=V_{t+1}(\theta-a ; 2(\theta-a)-(y-2 a))=V_{t+1}(\theta-a ; 2 \theta-y)
$$

where the second equation follows from applying property (9.1) to $V_{t+1}(\theta-a ; y-2 a)$. Using the two equations above, we obtain

$$
\begin{aligned}
F(\theta ; \theta+a) & =\int_{y: V_{t+1}(\theta+a ; y) \geq 0} V_{t+1}(\theta ; y) d y=\int_{y: V_{t+1}(\theta-a ; 2 \theta-y) \geq 0} V_{t+1}(\theta ; 2 \theta-y) d y \\
& =\int_{z: V_{t+1}(\theta-a ; z) \geq 0} V_{t+1}(\theta ; z) d z=F(\theta ; \theta-a),
\end{aligned}
$$

where the last equation follows from the change of variable $z=2 \theta-y$. Hence, $F$ is diagonally symmetric. 
Finally, we show that $V_{t}$ is strictly concave. Since

$$
V_{t}(\theta ; x)=\lambda_{t} u(\theta ; x)+V_{t+1}(\theta ; x)+F(\theta ; x)
$$

and $u(\theta ; \cdot), V_{t+1}(\theta ; \cdot)$ are strictly concave by induction assumption it suffices to show that the function $F(\theta ; \cdot)$ is concave for all $\theta$.

Using diagonal symmetry and anonymity of $F$, we obtain

$$
F(\theta ; x)=F(0, x-\theta)=F(0, \theta-x)=F(x, \theta)
$$

for all $\theta, x$. Similarly, $V_{t+1}(\theta ; x)=V_{t+1}(x ; \theta)$. Consequently,

$$
F(\theta ; x)=F(x ; \theta)=\int_{y: V_{t+1}(\theta ; y) \geq 0} V_{t+1}(x ; y) d y=\int_{y: V_{t+1}(\theta ; y) \geq 0} V_{t+1}(y ; x) d y .
$$

In order to prove concavity of $F(\theta ; \cdot)$, we show that

$$
F(\theta ; a x+(1-a) z) \geq a F(\theta ; x)+(1-a) F(\theta ; z)
$$

for all $\theta, x, z \in \Theta$ and all $a \in[0,1]$. By equation (9.3), we have

$$
\begin{aligned}
F(\theta ; a x+(1-a) z) & =\int_{y: V_{t+1}(\theta ; y) \geq 0} V_{t+1}(y ; a x+(1-a) z) d y \\
& \geq \int_{y: V_{t+1}(\theta ; y) \geq 0}\left(a V_{t+1}(y ; x)+(1-a) V_{t+1}(y ; z)\right) d y \\
& =a \int_{y: V_{t+1}(\theta ; y) \geq 0} V_{t+1}(y ; x) d y+(1-a) \int_{y: V_{t+1}(\theta ; y) \geq 0} V_{t+1}(y ; z) d y \\
& =a F(\theta ; x)+(1-a) F(\theta ; z) .
\end{aligned}
$$

Here, the inequality follows from the induction assumption that $V_{t+1}(y ; \cdot)$ is concave. This completes the proof of our statement.

(b) In order to prove that the special limit equilibrium $S$ is the almost unique limit equilibrium, we show that the game satisfies the indifference condition and the boundedness condition. Since $V_{t}$ is diagonally symmetric, anonymous and strictly concave for all $t$ we have that

$$
\left\{x \mid V_{t}(\theta ; x)=0\right\} \text { is either empty or equal to }\left\{\theta-a_{t}, \theta+a_{t}\right\}
$$

for all $t$ and $\theta$,where $a_{t}$ does not depend on $\theta$. Hence, $\left\{x \mid V_{t}(\theta ; x)=0\right\}$ always has Lebesgue measure zero, implying that the game satisfies the indifference condition. 
In order to prove the boundedness condition, let $a<0$ be given. Since $V_{t}$ is diagonally symmetric, anonymous and strictly concave, we have that

$$
\left\{x \mid V_{t}(\theta ; x) \geq a\right\} \text { is either empty or equal to }\left[\theta-b_{t}, \theta+b_{t}\right]
$$

for all $t$ and $\theta$ where $b_{t}$ does not depend on $\theta$. So, $\rho\left(\left\{x \mid V_{t}(\theta ; x) \geq a\right\}\right)=0$ or $2 b^{t}$ for all $t$ and $\theta$. Let $M=\max \left\{2 b_{1}, \ldots, 2 b_{T}\right\}$. Then, $\rho\left(\left\{x \mid V_{t}(\theta ; x) \geq a\right\} \leq M\right.$ for all $t$ and $\theta$ and hence the boundedness condition is satisfied.

(c) Next, we show that the limit equilibrium $S$ is such that $S_{t}(\theta) \subset\{x \mid u(\theta ; x) \geq 0\}$ for all $t$ and $\theta$. We use induction on $t$.

For $t=T$ the statement is true since $S_{T}(\theta)=\left\{x \mid V_{T}(\theta ; x) \geq 0\right\}=\left\{x \mid \lambda_{T} u(\theta ; x) \geq 0\right\}=$ $\{x \mid u(\theta ; x) \geq 0\}$ since $\lambda_{t}>0$.

Now let $t<T$. Suppose that $u(\theta ; x)<0$. We show that $V_{t}(\theta ; x)<0$.

Since by assumption $S_{t+1}=\left\{x \mid V_{t+1}(\theta ; x) \geq 0\right\} \subset\{x \mid u(\theta ; x) \geq 0\}$ it follows that $V_{t+1}(\theta ; x)<$ 0 . By definition,

$$
V_{t}(\theta ; x)=\lambda_{t} u(\theta ; x)+V_{t+1}(\theta ; x)+\int_{y: V_{t+1}(x ; y) \geq 0} V_{t+1}(\theta ; y) d y .
$$

As before, let

$$
F(\theta ; x)=\int_{y: V_{t+1}(x ; y) \geq 0} V_{t+1}(\theta ; y) d y .
$$

Therefore it suffices to show that $F(\theta ; x) \leq 0$. Since $V_{t+1}$ is strictly concave, anonymous and diagonally symmetric it follows that $\left\{y \mid V_{t+1}(x ; y) \geq 0\right\}$ is either empty or equal to $[x-a, x+a]$ for some $a \geq 0$.

If it is empty, then $F(\theta ; x)=0$ by definition. If $a=0$ then, also, $F(x)=0$. Now, suppose that $a>0$. Then,

$$
\begin{aligned}
F(\theta ; x) & =\int_{y \in[x-a, x+a]} V_{t+1}(\theta ; y) d y=2 a \int_{y \in[x-a, x+a]} V_{t+1}(\theta ; y) \frac{1}{2 a} d y \\
& =2 a E_{\mu}\left[V_{t+1}(\theta ; \cdot)\right]
\end{aligned}
$$

where $\mu$ is the uniform distribution on $[x-a, x+a]$ and $E_{\mu}\left[V_{t+1}(\theta ; \cdot)\right]$ is the expected value of $V_{t+1}(\theta ; \cdot)$ with respect to $\mu$. Since $V_{t+1}(\theta ; \cdot)$ is concave we know by Jensen's inequality that

$$
E_{\mu}\left[V_{t+1}(\theta ; \cdot)\right] \leq V_{t+1}\left(\theta ; E_{\mu}(y)\right)=V_{t+1}(\theta ; x)<0
$$

which implies that $F(x)<0$. This completes the proof of the statement.

(d) Finally, we turn to the special case where only the utility at the final stage is relevant, i.e. $\lambda_{t}=0$ for all $t<T$. We show that $S_{t}(\theta) \subset S_{t+1}(\theta)$ for all $t$ and $\theta$. 
In this special case, the value functions $V_{t}$ are given by the recursive formula $V_{T}(\theta ; x)=$ $\lambda_{T} u(\theta ; x)$ and

$$
V_{t}(\theta ; x)=V_{t+1}(\theta ; x)+\int_{y: V_{t+1}(x ; y) \geq 0} V_{t+1}(\theta ; y) d y=V_{t+1}(\theta ; x)+F(\theta ; x)
$$

if $t<T$. Suppose that $x \notin S_{t+1}(\theta)$. Then, by definition, $V_{t+1}(\theta ; x)<0$. In the proof of the previous statement, we have seen that $V_{t+1}(\theta ; x)<0$ implies $F(x)<0$. Hence, $V_{t}(\theta ; x)=$ $V_{t+1}(\theta ; x)+F(x)<0$ which means that $x \notin S_{t}(\theta)$. This completes the proof.

\section{References.}

1. Aumann, R.J. (1964). Markets with a Continuum of Traders. Econometrica 32, 39-50.

2. Barberà, S., Maschler, M. and J. Shalev (1998). Voting for Voters: A Model of Electoral Evolution. Working Paper.

3. Gomes, A., Hart, S. and A. Mas-Colell (1999). Finite Horizon Bargaining and the Consistent Field. Games and Economic Behavior 27, 204-228.

4. Kotter, J.P. (1979). "Power in Management". AMACOM, A Division of American Management Associations, New York.

5. Kreps, D. and E.L. Porteus (1979). Dynamic Choice Theory and Dynamic Programming. Econometrica 47, 91-100.

6. Schmeidler, D. (1973). Equilibrium Points of Nonatomic Games. Journal of Statistical Physics 7, 295-300.

7. Streufert, P.A. (1990). Stationary Recursive Utility and Dynamic Programming under the Assumption of Biconvergence. Review of Economic Studies 57, 79-97. 